\title{
Manager- und transaktionsspezifische Determinanten der Performance von Arbitrage CLOs
}

Julia Scholz

Discussion Paper 2011-01

5. Februar 2011

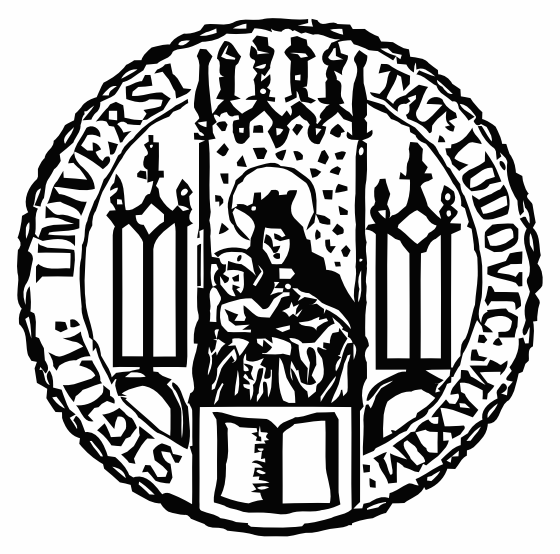

Munich School of Management

University of Munich

Fakultät für Betriebswirtschaft

Ludwig-Maximilians-Universität München

Online at http://epub.ub.uni-muenchen.de/ 


\title{
Manager- und transaktionsspezifische Determinanten der Performance von Arbitrage CLOs
}

\author{
Julia Scholz* \\ 5. Februar 2011
}

\begin{abstract}
Zusammenfassung
Der vorliegende Beitrag untersucht die Determinanten der Performance europäischer Arbitrage Collateralized Loan Obligations für das Jahr 2009. Der Fokus liegt dabei auf der Bedeutung der performanceabhängigen Vergütung des CLO-Managers, den Eigenschaften des CLO-Managers und der Transaktionscharakteristika als mögliche Einflussfaktoren der Rating Performance. Es wird gezeigt, dass Transaktionen, bei denen dem CLO-Manager eine Incentive Management Fee gewährt wird, mit einer höheren Wahrscheinlichkeit herabgestuft werden als Transaktionen ohne Incentive Fee. Dieser Befund bestätigt die Hypothese, dass durch die Incentive Fee Risikoanreize für CLO-Manager geschaffen werden. Des Weiteren wird ein positiver Zusammenhang zwischen der Erfahrung bzw. der Größe eines CLO-Managers und der Rating Performance festgestellt. Der Einfluss des Managers auf die Performance einer CLO-Transaktion wird auch an den weiteren in der Studie herangezogenen managerspezifischen Charakteristika wie Typ und Unternehmenssitz bestätigt. Für die Transaktionscharakteristika wird hingegen im betrachteten Untersuchungszeitraum kein signifikanter Einfluss auf die Rating Performance nachgewiesen.
\end{abstract}

Stichworte: Verbriefung, Collateralized Loan Obligations, Collateral Manager, CLO-Management, Anreize, Ratingherabstufungen

JEL Klassifikation: G10, G21, G24

* Institut für Kapitalmarktforschung und Finanzierung, Fakultät für Betriebswirtschaft, LudwigMaximilians-Universität München, Schackstr. 4, 80539 München. 


\section{Problemstellung}

In der seit Sommer 2007 dauernden Finanzkrise sind Verbriefungen jeglicher Art erheblich unter Druck geraten. Allein am europäischen Markt nahmen die Ratingagenturen 2008 rund 7.000 und 2009 rund 11.000 Ratingherabstufungen vor, während 2007 nur rund 800 Herabstufungen zu verzeichnen waren. ${ }^{1}$ Aufgrund der niedrigen Ausfallraten in den Jahren vor der Finanzkrise sind Unterschiede in der Performance von Verbriefungstransaktionen auf dem noch relativ jungen europäischen Markt erst seit Mitte 2008 zu erkennen. In der Literatur existieren bislang nur wenige Studien, die sich mit der Performance von Verbriefungen und ihren Bestimmungsfaktoren befassen. Dies ist insbesondere mit der am Markt fehlenden Preistransparenz zu begründen. Zudem weisen die Transaktionen vielfältige Ausgestaltungsformen auf, die einen Performancevergleich vor allem über die verschiedenen Marktsegmente hinweg erschweren. ${ }^{2}$ Ziel der vorliegenden Studie ist es, die bislang kaum erforschten Qualitätseinflüsse typischer Kreditrisikotransferprodukte durch eine empirische Analyse der Erklärungsdeterminanten der Performance europäischer Arbitrage Collateralized Loan Obligations (Arbitrage CLOs) herauszuarbeiten. Der Fokus liegt dabei auf den spezifischen Gestaltungsmerkmalen dieser Transaktionen.

Arbitrage CLOs sind eine Untergruppe der Asset Backed Securities (ABS). Unter ABS werden festverzinsliche Wertpapiere oder Schuldscheine verstanden, die Zahlungsansprüche gegen eine eigens zum Zweck der Transaktion gegründeten Zweckgesellschaft verbriefen. Sie werden durch ein Portfolio von Vermögenswerten (Assets) gedeckt, die auf die Zweckgesellschaft übertragen wurden und den Inhabern der ABS als Haftungsmasse zur Verfügung stehen. Im Fall von Collateralized Loan Obligations (CLOs) bezieht sich das Portfolio auf Unternehmenskredite, üblicherweise Leveraged Loans. ${ }^{3}$ Bei einer Arbitrage CLO-Transaktion erwirbt ein Asset Manager (CLO-Manager oder Collateral Manager) im Auftrag der Zweckgesellschaft Kreditforderungen, die von der Zweckgesellschaft zu einem Pool zusammengefasst und in Form von Wertpapieren am Markt platziert werden. Der Asset Manager tritt also an die Stelle des Originators einer Balance Sheet CLO-Transaktion, bei der der Originator ein bestehendes Kreditportfolio aus seiner Bilanz veräußert. ${ }^{4}$

Dem CLO-Manager kommt für die Performance der von ihm verwalteten Transaktion eine zentrale Rolle zu, da er neben der Auswahl der Kreditforderungen auch die Überwachung der Wertentwicklung übernimmt und ein aktives Management der Portfoliobe-

1 Vgl. European Securitisation Forum (2008), S. 11, und European Securitisation Forum (2009), S. 11.

2 Hierzu zeigen Adelson u.a. (2002), Adelson / Bartlett (2005) und Newman u. a. (2008), dass sich die Ausfall- und Downgradehäufigkeiten in den verschiedenen Marktsegmenten des US-amerikanischen Verbriefungsmarktes stark unterscheiden.

3 Für einen Überblick über die Segmente des ABS-Marktes siehe Jobst (2002), S. 8.

${ }^{4}$ Für eine ausführlichere Differenzierung zwischen Arbitrage CLOs und Balance Sheet CLOs siehe Schiefer (2008), S. 204-224. 
standteile betreibt. ${ }^{5}$ Zur Herausarbeitung der Performancedeterminanten von Arbitrage CLOs werden daher in der vorliegenden Untersuchung neben transaktionsabhängigen Charakteristika die performanceabhängige Vergütung des CLO-Managers sowie weitere managerspezifische Eigenschaften herangezogen. Zu den managerspezifischen Eigenschaften zählen dabei die Erfahrung, die Anzahl bzw. das Volumen der vom Manager insgesamt durchgeführten CLO-Transaktionen (seine „Größe“), der Typ und der Unternehmenssitz. Der besondere Grund der Einbeziehung der Managervergütung in die Untersuchung ist darin zu sehen, dass es in gemanagten Kreditverbriefungstransaktionen zu Moral HazardProblemen auf Seiten des CLO-Managers kommen kann, deren Bedeutung im Wesentlichen von der vertraglichen Gestaltung der Vergütung abhängt. ${ }^{6}$ Da die beim Management von CLO-Transaktionen auftretenden Anreizprobleme in der Literatur bislang nur theoretisch (Garrison 2005) und auf Basis eines Simulationsmodells (Scholz 2009) untersucht worden sind, ist meines Wissens die vorliegende Studie die erste empirische Analyse, die sich mit den Anreizproblemen von CLO-Managern befasst.

In die Untersuchung werden sämtliche zwischen September 1999 und Juni 2008 emittierten Transaktionen einbezogen. Die Performance wird anhand der Ratingherabstufungen der Ratingagenturen Moody's, Standard \& Poor's und Fitch gemessen. Hierbei werden lediglich die Ratingherabstufungen im Jahr 2009 herangezogen, da die Agenturen als Konsequenz aus der im Zuge der Finanzkrise eingetretenen Verschlechterung der Kreditqualität vieler Schuldner Anfang des Jahres 2009 begonnen haben, ihre Annahmen und Methoden zur Bewertung von CLOs zu überarbeiten und sämtliche CLO-Ratings zu überprüfen, was drastische Korrekturen der Wertpapierratings zur Folge hatte. Daher werden aus Gründen der Vergleichbarkeit Ratingherabstufungen vor 2009 aus der Betrachtung ausgeblendet und der Untersuchungszeitraum auf das Jahr 2009 beschränkt. Die Bedeutung der ausgewählten manager- und transaktionsspezifischen Charakteristika als Determinanten zur Erklärung der Rating Performance wird über einen Probit-Regressionsansatz geprüft, der als abhängige Variable die Wahrscheinlichkeit einer Ratingherabstufung verwendet.

Die Bedeutung der durch die Vergütung hervorgerufenen Leistungs- und Risikoanreize von CLO-Managern bei Arbitrage CLO-Transaktionen wird durch die Subordinated Fee und die Incentive Fee, die beiden variablen Bestandteile der Managervergütung, untersucht. Von diesen beiden Vergütungskomponenten wird lediglich für letztere ein Einfluss auf die Rating Performance nachgewiesen. So ist die Wahrscheinlichkeit einer Ratingherabstufung für Transaktionen höher, wenn dem CLO-Manager eine Incentive Fee gewährt wird. Dieses Ergebnis weist darauf hin, dass die Incentive Fee für CLO-Manager Anreize zu einer risikoreichen Anlagestrategie schafft. Mit der variablen Vergütung zusammenhängende po-

5 Vgl. Moody's Investors Service (2010), S. 2, Standard \& Poor's (2009a), S. 30, und Fitch (2008a), S. 12-13.

6 Vgl. Keller (2008), S. 3, und Ashcraft / Schuermann (2008), S. 9-10. 
sitive Leistungsanreizeffekte werden dagegen nicht nachgewiesen. Der Einfluss eines CLOManagers auf die Performance der von ihm verwalteten Transaktion wird auch anhand der weiteren in der Studie verwendeten managerspezifischen Charakteristika bestätigt. Für die Erfahrung und die Größe eines CLO-Managers wird ein negativer Zusammenhang mit der Wahrscheinlichkeit einer Ratingherabstufung festgestellt. In Bezug auf den Typ eines Managers zeigt sich, dass von Investmentbanken gemanagte Transaktionen im Untersuchungszeitraum mit einer geringeren Wahrscheinlichkeit herabgestuft werden als Transaktionen, bei denen ein Multi-Asset Manager, ein Hedge Fond oder eine Private Equity-Gesellschaft als CLO-Manager fungiert. Schließlich weisen Transaktionen US-amerikanischer CLOManager eine im Durchschnitt geringere Downgrade-Wahrscheinlichkeit auf als Transaktionen europäischer Manager. Dieses Ergebnis deutet darauf hin, dass US-amerikanische Manager aufgrund ihrer Managementtätigkeiten auf dem amerikanischen Markt über eine höhere Expertise im Management von CLO-Transaktionen verfügen als europäische Manager. Für die herangezogenen transaktionsspezifischen Charakteristika wird im zugrunde gelegten Untersuchungszeitraum dagegen kein Einfluss auf die Rating Performance nachgewiesen.

Die vorliegende Studie ist wie folgt aufgebaut: In Kapitel 2 wird zunächst ein kurzer Überblick über die Ergebnisse der einschlägigen Literatur gegeben. Anschließend werden in Kapitel 3 die Hypothesen zu den Erklärungsdeterminanten der Rating Performance gemanagter Kreditverbriefungstransaktionen hergeleitet. Die empirische Untersuchung erfolgt in Kapitel 4, bevor dann der Beitrag mit einer kurzen Zusammenfassung und einem Ausblick in Kapitel 5 schließt.

\section{Stand der Literatur}

Zur Performance von Verbriefungen und deren Einflussfaktoren liegen bisher nur wenige Studien vor. Adelson u. a. (2002) und Adelson / Bartlett (2005) untersuchen im Rahmen einer deskriptiven Analyse die Ratingherabstufungen und Ausfälle von Asset Backed Securities im engeren Sinne (i. e. S.) am US-amerikanischen Verbriefungsmarkt für die Jahre 1990 bis Mitte 2001 bzw. bis Mitte 2004. ${ }^{7}$ Sie finden Unterschiede in den Downgrade- und Ausfallhäufigkeiten von ABS-Transaktionen in Abhängigkeit von der Art der zugrunde liegenden Assets, dem Emissionsjahrgang und der Ratingagentur, die eine Transaktion bewertet. Gleichermaßen zeigen Newman u.a. (2008) anhand einer deskriptiven Analyse der Ratingherabstufungen US-amerikanischer Collateralized Debt Obligations (CDOs)

7 Der Gruppe der ABS i. e. S. werden z. B. Verbriefungen von Kreditkartenforderungen, von Studentenkrediten, von Automobilratenkrediten oder von Konsumentenforderungen zugeordnet. Siehe Jobst (2002), S. 8. 
für die Jahre 1997 bis 2007, dass die Downgraderaten und -intensitäten der verschiedenen CDO-Klassen und Emissionsjahrgänge stark variieren.

Vergleichbar mit der vorliegenden Studie, deren Fokus auf der Rolle des Asset Managers in gemanagten CLO-Transaktionen liegt, untersuchen Cantor / Hu (2007), Keys u. a. (2009) und Güttler / Hommel / Reichert (2010) den Einfluss verschiedener bei statischen Verbriefungen beteiligter Parteien auf die Transaktionsperformance. ${ }^{8}$ Cantor / Hu (2007) analysieren den Einfluss bestimmter Charakteristika des Sponsors auf die Performance US-amerikanischer Asset Backed Securities i. e.S. und Mortgage Backed Securities, die im Zeitraum von 1993 bis 2002 emittiert wurden. ${ }^{9}$ Sie stellen einen signifikanten Einfluss des Typs und des Ratings des Sponsors auf die Wahrscheinlichkeit einer Ratingherabstufung bzw. Wertminderung der Wertpapiere fest, wenn die Rating Performance über die Asset-Klassen hinweg analysiert wird. Wird jedoch für die Art der zugrunde liegenden Vermögenswerte kontrolliert, ist nur noch ein geringer Einfluss des Sponsortyps auf die Performance zu beobachten. Dies deutet darauf hin, dass die festgestellten Performanceunterschiede durch eine ungleichmäßige Verteilung der verschiedenen Sponsortypen über die Asset-Klassen hervorgerufen wurden. Keys u. a. (2009) untersuchen den Einfluss bestimmter Charakteristika von Originatoren auf die Performance verbriefter Kredite am US-amerikanischen Subprime-Markt in den Jahren 2001 bis 2006. Sie finden, dass die Wahrscheinlichkeit eines Kreditausfalls mit der Größe, dem Anteil liquider Assets und dem Anteil der Sichteinlagen an der Bilanzsumme der verbriefenden Bank sinkt. Dies weist darauf hin, dass Originatoren mit einer höheren Qualität und Liquidität ein sorgfältigeres Screening bei der Kreditvergabe durchführen. Des Weiteren analysieren sie die Bedeutung möglicher auf Seiten des Originators bestehender Anreizprobleme bei der Kreditvergabe. Hierbei stellen sie einen negativen Einfluss der an der Vergütung gemessenen relativen Macht des Risikomanagers des Originators auf die Wahrscheinlichkeit eines Kreditausfalls fest, während sie für die Höhe der Vergütung des Top Management des Originators keinen Zusammenhang mit der Performance der Kredite nachweisen können. Diesen Befund führen sie darauf zurück, dass Moral Hazard-Probleme schwächer ausgeprägt sind, wenn die Risiko Management-Abteilung des Originators eine stärkere Verhandlungsmacht besitzt. Güttler / Hommel / Reichert (2010) analysieren die Bedeutung der Eigenschaften des Sponsors, des Servicers und des Underwriters als Erklärungsdeterminanten der Performance US-amerikanischer Residential Mortgage Backed Securities für die Jahre 2000 bis 2003, wobei sie die Performance einer Transaktion anhand der in den drei Jahren nach Closing durchschnittlichen monatlichen Rückstandsquote der im zugrunde liegenden Asset Pool befindlichen Hypothekenforderungen messen. Sie beobachten, dass Asset Pools von großen und finanziell gesunden Sponsoren im Durchschnitt eine bessere Performance

\footnotetext{
8 Für die beteiligten Parteien einer Verbriefungstransaktion und ihre Funktionen siehe Waschbusch (1998), S. 409-414, Kothari (2006), S. 201-202, und Schiefer (2008), S. 71-80.

9 Als Untersuchungszeitraum verwenden sie die Ratingänderungen bis Juni 2006.
} 
aufweisen. Ebenso stellen sie für die Größe und die Bonität des Servicers einen positiven Zusammenhang mit der Performance des Pools fest. Diese Befunde weisen darauf hin, dass Institutionen mit einer längeren Marktperspektive aufgrund von Reputationsbedenken weniger dazu neigen, Informationsasymmetrien zur Erzielung kurzfristiger Gewinne auszunutzen. Im Widerspruch dazu finden sie einen negativen Zusammenhang zwischen dem Marktanteil des Underwriters und der Performance des Asset Pools. Diesen Befund führen sie darauf zurück, dass Investmentbanken mit einem hohen Marktanteil im Verbriefungsgeschäft ihre Reputation zur Steigerung kurzfristiger Gewinne genutzt haben.

Die Anreizprobleme bei gemanagten Verbriefungstransaktionen sind bislang nur Gegenstand der Untersuchungen von Garrison (2005), Keller (2008) und Scholz (2009). Garrison (2005) analysiert auf Basis eines theoretischen Modells das Leistungs- und das Risikoanreizproblem bei gemanagten CDO-Transaktionen und wägt die damit verbundenen Agency-Kosten für verschiedene Formen der Beteiligung des Asset Managers an der von ihm verwalteten Transaktion gegeneinander ab. In Übereinstimmung mit dem Befund von Innes (1990), dass das Halten einer Eigenkapitalposition für einen beschränkt haftenden Agenten optimal ist, zeigt Garrison, dass eine Beteiligung des Managers an der Equity Tranche im Hinblick auf die Leistungsanreizwirkung ex ante effizient ist. Allerdings besitzt der Manager unter Vernachlässigung von Transaktionskosten immer dann einen Anreiz zur Steigerung des Portfoliorisikos, wenn, in absoluten Werten gemessen, seine Beteiligung an der Equity Tranche seine Beteiligung an den Debt Tranchen übersteigt. ${ }^{10}$ Insgesamt jedoch überwiegen bereits bei einer geringen Effektivität der Screeninganstrengung die mit einer Eigenkapitalbeteiligung verbundenen positiven Leistungsanreizeffekte die Agency-Kosten des Risk Shifting. Keller (2008) identifiziert das Leistungs- und das Risikoanreizproblem als mögliche Probleme asymmetrischer Informationsverteilung zwischen dem Asset Manager einer CLO-Transaktion und den Investoren der emittierten Tranchen und legt auf Basis einer qualitativen Analyse dar, wie sich diese Probleme auf die Strategien des CLO-Managers in Bezug auf die Verwaltung des verbrieften Portfolios auswirken. Im Rahmen einer Darstellung der in der Praxis zu findenden Mechanismen zur Lösung der Anreizprobleme untersucht Keller mittels einer empirischen Analyse die Erklärungsdeterminanten für die Vereinbarung einer Bestimmung, die den CLO-Manager zum Kauf und gegebenenfalls Halten eines bestimmten Anteils an der Equity Tranche verpflichtet. Hierbei beobachtet er, dass die Wahrscheinlichkeit der Vereinbarung einer Haltepflicht mit der Größe der CLO-Transaktion sowie der Erfahrung des CLO-Managers steigt. Scholz (2009) betrachtet das Risikoverhalten von CLO-Managern isoliert von deren Screening- und Monitoringanreizen. Sie untersucht im Rahmen einer Simulationsstudie, wie ein CLO-Manager die Auswahl der der Transaktion zugrunde liegenden Kredite in

${ }^{10}$ Als Debt Tranchen werden hierbei die gegenüber der Equity Tranche vorrangigen Tranchen (Senior und Mezzanine Tranchen) bezeichnet. 
Bezug auf deren Ausfallwahrscheinlichkeit, Konzentration und Wiedergewinnungsquote trifft, wenn er seine Vergütung und, falls vorhanden, den Wert seiner Beteiligung an der Equity Tranche maximiert. Sie zeigt, dass die Incentive Fee und die Beteiligung an der Equity Tranche einen positiven Zusammenhang mit dem Risiko des Asset Pools aufweisen und daher für den CLO-Manager Anreize zur Gestaltung eines möglichst riskanten Portfolios setzen. Die Senior Fee und die Subordinated Fee sinken hingegen mit steigendem Risiko des verbrieften Portfolios im Wert und wirken somit gegen ein Risk Shifting des Managers. Insgesamt ergibt sich, dass der Manager stets einen Anreiz zur Maximierung der Portfoliokonzentration besitzt. Die Ausfallwahrscheinlichkeit und Wiedergewinnungsquote der Kredite maximiert bzw. minimiert er hingegen nur dann, wenn der Anteil der Incentive Fee an der Gesamtvergütung hoch ist oder eine gewisse Managerbeteiligung an der Equity Tranche vorliegt.

Die vorliegende Studie unterscheidet sich von den bisherigen Untersuchungen vor allem dadurch, dass sie (1) die Determinanten der Performance von Arbitrage CLOs analysiert, (2) den europäischen Verbriefungsmarkt für die Untersuchung heranzieht und (3) die Rolle des Asset Managers in gemanagten Kreditverbriefungen untersucht. Der Literaturüberblick zeigt, dass damit eine nicht unerhebliche Forschungslücke geschlossen werden kann.

\section{Hypothesen zu den Erklärungsdeterminanten der Performance von Arbitrage CLO-Transaktionen}

\subsection{Messung der Performance anhand von Ratingherabstufungen}

Die Performance von Arbitrage CLOs wird in der vorliegenden Studie über die Ratingherabstufungen der drei großen Ratingagenturen Moody's, Standard \& Poor's und Fitch gemessen. Idealerweise sollte die Performancemessung anhand von Renditen vorgenommen werden. Da für Verbriefungstransaktionen aufgrund ihres außerbörslichen Handels in der Regel keine transparenten Marktpreise verfügbar sind, wird auf Ratingänderungen zurückgegriffen. Da die Ratingagenturen zur Bonitätsbeurteilung nicht nur das Ausfallrisiko des zugrunde liegenden Asset Pools heranziehen, sondern auch die gesamte strukturelle und rechtliche Ausgestaltung der Transaktion, stellen Ratings das beste verfügbare Maß für die Kreditqualität einer Tranche dar. ${ }^{11}$ Bei der Verwendung von Ratingänderungen als

\footnotetext{
${ }^{11}$ Hierbei ist zu berücksichtigen, dass sich das Rating lediglich auf die Bonität einer Tranche bezieht, nicht aber auf andere den Marktpreis beeinflussende Risikofaktoren wie z. B. die Liquidität einer Tranche. Zur Ratingmethodik von CDOs, denen Arbitrage CLOs als Teilsegment zugeordnet sind, siehe Fender / Kiff (2004).
} 
Performancemaß ist zu berücksichtigen, dass Ausfälle im zugrunde liegenden Kreditpool nicht zwangsläufig zu Ratingherabstufungen der emittierten Tranchen führen. Besteht für die Tranchen ein ausreichendes Sicherheitenniveau, führen Ausfälle erst ab einer bestimmten Höhe zu Ratingherabstufungen.

Die Untersuchung des Einflusses der herangezogenen Erklärungsdeterminanten auf die Performance von Arbitrage CLOs wird auf Transaktionsebene durchgeführt. Als Maßs für die Performance einer Transaktion wird eine Dummyvariable verwendet, die den Wert Eins annimmt, wenn mindestens eine Tranche der Transaktion im Jahr 2009 herabgestuft wurde, und andernfalls den Wert Null. ${ }^{12}$ Anhand eines Probit-Regressionsmodells wird der Einfluss möglicher durch die Managervergütung hervorgerufener Anreizeffekte sowie bestimmter weiterer manager- und transaktionsspezifischer Charakteristika auf die Wahrscheinlichkeit einer Ratingherabstufung analysiert. Das für die Untersuchung herangezogene Modell ist allgemein durch folgende Gleichung beschrieben:

$$
\begin{gathered}
\text { Abhängige Variable }=f(\text { Vergütungsvariablen, managerspezifische Variablen, } \\
\text { transaktionsspezifische Variablen }) .
\end{gathered}
$$

Die Forschungsfragen der vorliegenden Studie lassen sich also grob in drei Bereiche einordnen. Der erste Bereich befasst sich mit der Frage, ob und gegebenenfalls in welchem Ausmaß sich mögliche Anreizprobleme auf Seiten des CLO-Managers auf die Performance einer Transaktion auswirken. Der zweite Bereich setzt sich mit der Bedeutung bestimmter Eigenschaften des CLO-Managers als mögliche Einflussfaktoren der Performance auseinander, der dritte Bereich mit der Bedeutung bestimmter Transaktionscharakteristika.

\subsection{Die Bedeutung von Anreizproblemen auf Seiten des CLO-Managers}

Die Anreize eines CLO-Managers bei der Verwaltung einer Transaktion werden insbesondere durch dessen Vergütung bestimmt. Hierbei ist zu berücksichtigen, dass sich die Stärke der Leistungs- und Risikoanreize eines CLO-Managers während der Laufzeit der Transaktion in Abhängigkeit der Wertentwicklung des Referenzportfolios ändern kann. Garrison (2005) zeigt in diesem Zusammenhang, dass für die Stärke der beiden Anreizeffekte die Wahrscheinlichkeit entscheidend ist, mit der die Position des CLO-Managers im Geld en-

\footnotetext{
${ }^{12}$ Aufgrund der Dummykodierung werden Transaktionen mit unterschiedlich herabgestuften Tranchentypen (Senior versus Mezzanine Tranchen), mit unterschiedlich starken Ratingherabstufungen und mit einer unterschiedlichen Anzahl an Ratingherabstufungen im Jahr 2009 in einer Kategorie zusammengefasst, so dass Unterschiede zwischen diesen Gruppen nicht Gegenstand der folgenden Untersuchung sind.
} 
det. Ist die vom Manager gehaltene Position aufgrund unerwartet hoher bzw. niedriger Verluste im unterlegten Asset Pool weit aus dem Geld bzw. weit im Geld, sind mit ihr keine oder nur geringe Leistungs- und Risikoanreize verbunden. Eine starke Anreizwirkung wird hingegen dann geschaffen, wenn die Position des Managers gerade im Geld liegt. ${ }^{13}$ Da in der vorliegenden Studie eine statische Analyse der Rating Performance von Arbitrage CLOs durchgeführt wird, werden die Hypothesen über die Wirkungszusammenhänge zwischen der Rating Performance und den durch die Vergütung induzierten Anreizeffekten für eine ex ante-Betrachtung abgeleitet.

Die in Arbitrage CLO-Transaktionen vorfindbaren Formen der Managervergütung sind vielfältig, wobei jedoch eine gemeinsame Grundstruktur zu erkennen ist. Typischerweise setzt sich diese aus drei Komponenten zusammen, der Senior Management Fee, der Subordinated Management Fee und der Incentive Management Fee. ${ }^{14}$ Die Managementgebühren werden aus den eingehenden Zahlungen des Asset Pools beglichen, wobei sie wie die Tranchen einer Transaktion eine bestimmte Priorität in Bezug auf die Verteilung der Cashflows besitzen. Die Senior Fee hat gegenüber sämtlichen Tranchen einer Transaktion einen übergeordneten Anspruch auf die Zahlungen des Asset Pools. Sie stellt die fixe Komponente der Vergütung eines CLO-Managers dar, da sie auch bei einer niedrigen Poolqualität vollkommen sicher ist. ${ }^{15}$ Von ihr geht keine bzw. eine zu vernachlässigende Anreizwirkung aus, weshalb die Senior Fee im Folgenden nicht weiter betrachtet wird. $\mathrm{Zu}$ den variablen und somit anreizwirksamen Vergütungsbestandteilen zählen die Subordinated Fee und die Incentive Fee. Die Subordinated Fee besitzt gegenüber den gerateten Tranchen (Senior und Mezzanine Tranchen) eine nachrangige Stellung und wird erst nach Bedienung der Zahlungsansprüche der Investoren dieser Tranchen, jedoch vor Bedienung der Ansprüche der Investoren der Equity Tranche gezahlt. Sie wird üblicherweise als jährlicher Prozentsatz definiert, der sich auf den Nominalwert des Asset Pools am Berechnungstag oder den in der Berechnungsperiode durchschnittlich vorhandenen Nominalwert des Pools bezieht. Ihr Risikoprofil ist daher vergleichbar mit dem einer nachrangigen Mezzanine Tranche. Fender / Mitchell (2009) zeigen anhand einer modelltheoretischen Analyse, dass durch den Einbehalt einer Mezzanine Tranche für den Originator, der in einer statischen Kreditverbriefungstransaktion das Screening und in der Regel auch das Monitoring der Kredite übernimmt, positive Leistungsanreize geschaffen werden, deren Stärke von der Qualität des Forderungspools, der Größe der Equity Tranche und den Konjunkturerwar-

\footnotetext{
${ }^{13}$ Vgl. Garrison (2005), S. 19-21.

${ }^{14} \mathrm{Im}$ Folgenden werden die drei Vergütungskomponenten mit Senior Fee, Subordinated Fee bzw. Incentive Fee abgekürzt. Für die Vergütungskomponenten sind auch andere Bezeichnungen zu finden. So wird beispielsweise die Senior Fee in einigen Fällen auch als „Management Fee“ oder „Base Fee“ bezeichnet, die Subordinated Fee auch als „Performance Fee“ oder „Junior Management Fee“ und die Incentive Fee auch als "Contingent Management Fee“ oder als "Second Subordinated Fee“.

${ }^{15}$ Vgl. Scholz (2009), S. 30.
} 
tungen des Originators abhängt. ${ }^{16}$ In einer dynamischen Kreditverbriefungstransaktion ist der CLO-Manager für das Screening und das Monitoring der zugrunde liegenden Forderungen verantwortlich. Nach dem Befund von Fender / Mitchell (2009) sollte daher von der Subordinated Fee eine positive Leistungsanreizwirkung für den CLO-Manager ausgehen, die ceteris paribus mit der Höhe dieser Vergütungskomponente ansteigt. Bezüglich des Risikoverhaltens des CLO-Managers einer Kreditverbriefungstransaktion zeigt Scholz (2009), dass der Wert der Subordinated Fee einen negativen Zusammenhang mit dem Risiko des Asset Pools aufweist und daher gegen mögliche Risk Shifting-Anreize des Managers wirkt. Hierbei ist der mit der Subordinated Fee verbundene Anreiz des CLO-Managers zur Gestaltung eines geringen Portfoliorisikos umso stärker, je höher diese Vergütungskomponente ist. Zusammenfassend ist daher zu vermuten, dass sowohl das Leistungs- als auch das Risikoanreizproblem durch die Ausgestaltung einer Subordinated Fee abgemildert werden und die Höhe der Subordinated Fee sich negativ auf die Wahrscheinlichkeit einer Ratingherabstufung einer CLO-Transaktion auswirkt.

Hypothese 1: Die Wahrscheinlichkeit einer Ratingherabstufung sinkt mit der Höhe der Subordinated Fee.

Die Incentive Fee besitzt im Vergleich zu den anderen beiden Vergütungskomponenten die geringste Priorität in Bezug auf die Bedienung aus den eingehenden Zahlungen des Asset Pools. Ihre Auszahlung ist in der Regel von der Erreichung einer „Incentive Fee Threshold" abhängig, die als jährliche Mindestrendite (Internal Rate of Return) der Equity Tranche definiert wird. Bezüglich der bei Überschreitung der Incentive Fee Threshold an den CLO-Manager fließenden Zahlungen sind zwei typische Formen zu beobachten: Entweder wird dem Manager ein bestimmter Anteil der bei Erreichung der Mindestrendite überschüssigen Zahlungen gewährt, die sonst an die Investoren der Equity Tranche fließen würden, oder er erhält eine jährliche Zahlung, die als Prozentsatz des Nominalwerts des Asset Pools definiert wird. Bis Erreichung der für die Equity Tranche vereinbarten Mindestrendite hat die Incentive Fee einen gegenüber der Equity Tranche untergeordneten Anspruch auf die Zahlungen des Pools. Wird die Incentive Fee Threshold erreicht, verfügt sie über die gleiche Priorität wie die Equity Tranche. Sie besitzt folglich Eigenkapitalcharakter, so dass die von Garrison (2005) und Fender / Mitchell (2009) in Bezug auf die Leistungsanreizwirkung der Equity Tranche ermittelten Ergebnisse auf die Incentive Fee angewandt werden können. Nach diesen sollte die Incentive Fee starke Leistungsanrei-

\footnotetext{
${ }^{16}$ Im Speziellen legen Fender / Mitchell dar, dass die erzielte Leistungsanreizwirkung vergleichsweise hoch ist, wenn die Qualität des Forderungspools niedrig, die Größe der Equity Tranche gering und die Wahrscheinlichkeit eines wirtschaftlichen Abschwungs hoch ist. Siehe Fender / Mitchell (2009), S. 17-19.
} 
ze für den CLO-Manager schaffen. ${ }^{17}$ Deshalb ist ceteris paribus davon auszugehen, dass die Screening- und Monitoringanstrengungen eines CLO-Managers, der eine Incentive Fee erhält, höher sind als die eines Managers, dem keine Incentive Fee gewährt wird. Die Existenz einer Incentive Fee sollte sich demnach negativ auf die Wahrscheinlichkeit einer Ratingherabstufung auswirken. Jedoch werden gemäß Scholz (2009) durch die Incentive Fee auch Anreize für den CLO-Manager geschaffen, das Risiko des Referenzportfolios zu steigern, womit eine höhere Downgrade-Wahrscheinlichkeit der Transaktion verbunden ist. Die beiden durch die Incentive Fee hervorgerufenen Anreizeffekte wirken sich folglich gegensätzlich auf die Performance einer Arbitrage CLO-Transaktion aus. Die Wirkungsrichtung der Incentive Fee hängt davon ab, welcher der beiden Effekte dominiert. Der Einfluss auf die Wahrscheinlichkeit einer Ratingherabstufung ist dann positiv (negativ), wenn der Risikoanreizeffekt den Leistungsanreizeffekt überwiegt (unterliegt).

Hypothese 2: Die Wirkungsrichtung der Incentive Fee auf die Wahrscheinlichkeit einer Ratingherabstufung bestimmt sich in Abhängigkeit des dominierenden Anreizeffekts.

\subsection{Die Bedeutung von Eigenschaften des CLO-Managers}

Dem Asset Manager einer Arbitrage CLO-Transaktion obliegt eine Reihe von Aufgaben. ${ }^{18}$ Er erwirbt die Kredite des Referenzportfolios zu Beginn der Transaktion, reinvestiert die während der Laufzeit eingehenden Tilgungszahlungen und tauscht innerhalb eines jährlich begrenzten Handelsvolumens Kredite des Pools gegen neue Kredite aus mit dem Ziel, Handelsgewinne zu erzielen. Hierbei muss er im Treuhandvertrag (Indenture) festgelegte Vorgaben in Bezug auf die Zusammensetzung, die Qualität und die Diversifikation des Referenzportfolios einhalten. Zudem ist er für die Überwachung der Bonität der einzelnen Referenzschuldner sowie der Qualität des gesamten Portfolios zuständig. Häufig übernimmt er auch die Bearbeitung und Abwicklung notleidend gewordener Kreditengagements (Workout-Management). Aufgrund der ihm obliegenden Aufgaben kann der CLO-Manager einen erheblichen Einfluss auf die Performance einer Transaktion haben. ${ }^{19}$ So stellt die Prüfung der Fähigkeiten eines CLO-Managers auch einen wichtigen Bestandteil der Beurteilung der Ratingagenturen dar. Für die Fähigkeiten eines CLO-Managers

\footnotetext{
${ }^{17}$ In diesem Zusammenhang finden auch Gan / Mayer (2007) bei ihrer Untersuchung des Verhaltens von Servicern am US-amerikanischen Markt für Commercial Mortgage Backed Securities, dass Servicer notleidende Kredite effizienter abwickeln, wenn sie die Erstverlusttranche halten.

${ }^{18}$ Zu den Aufgaben des Asset Managers einer Kreditverbriefungstransaktion siehe auch Standard \& Poor's (2002), S. 6 und S. 56-62, Braun / Schmidt (2005), S. 207, und Fitch (2007), S. 11-17.

19 Siehe Moody's Investors Service (2010), S. 2, Standard \& Poor's (2009a), S. 30, und Fitch (2008a), S. 12-13. In diesem Zusammenhang ist auch die Studie von Lassalvy / Lautard (2010) zu nennen, die für die 2009 am Markt befindlichen CLO-Manager große Unterschiede in den Ausfallraten der verwalteten Portfolios feststellen und diesen Tatbestand auf die unterschiedlichen Managementqualitäten zurückführen. Siehe Lassalvy / Lautard (2010), S. 15.
} 
sollten insbesondere dessen Erfahrung und Größe von Bedeutung sein. ${ }^{20}$ CLO-Manager mit langjähriger Markterfahrung haben aufgrund ihrer Kontakte auf dem Primär- und Sekundärmarkt einen guten und zeitnahen Zugang zu Informationen und sollten daher besser als neue Manager in der Lage sein, hochwertige Vermögenswerte zu akquirieren, ein breit diversifiziertes Portfolio zusammenzustellen und dieses während der Laufzeit aufrecht zu erhalten. Gleichermaßen sollte sich die Erfahrung eines Managers im Workout notleidender Kredite positiv auf die dabei zu erzielenden Ergebnisse und folglich auf die Performance der CLO-Transaktion auswirken. In Bezug auf die Größe eines CLO-Managers ist davon auszugehen, dass größere Manager über eine stärkere Verhandlungsposition beim Kauf und Verkauf von Vermögenswerten verfügen und besser ihre Preise als kleinere Manager durchsetzen können. Daneben besitzen größere Asset Manager in der Regel höhere und vermutlich bessere Research- sowie Workout-Kapazitäten. Dies motiviert

Hypothese 3: Die Wahrscheinlichkeit einer Ratingherabstufung sinkt mit der Erfahrung und der Größe des CLO-Managers.

\subsection{Die Bedeutung transaktionsspezifischer Charakteristika}

Die Bonitätseinstufungen der Ratingagenturen werden zu einem erheblichen Maß durch die Qualität des einer CLO-Transaktion zugrunde liegenden Portfolios bestimmt. ${ }^{21}$ Jedoch spielen auch die Tranchierung und die vereinbarten Sicherungsmaßnahmen (Credit Enhancement) eine bedeutende Rolle für die Bewertung der Tranchen. ${ }^{22}$ So ist es beispielsweise möglich, dass zwei Tranchen mit gleichem Attachment und Detachment Point, jedoch mit qualitativ unterschiedlichen Referenzportfolios, dasselbe Rating erhalten, wenn die Tranche der Transaktion mit dem Portfolio von geringerer Qualität mit höheren Credit

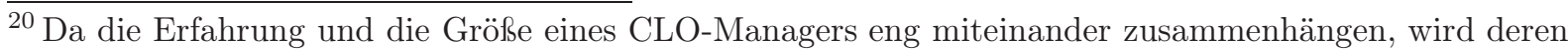
Einfluss gemeinsam und nicht getrennt voneinander betrachtet. Gleichzeitig lässt sich die Erfahrung und die Größe eines Managers auch in Zusammenhang mit dessen Reputation bringen, die möglichen Leistungs- und Risikoanreizproblemen auf Seiten des Managers entgegenwirken kann. Vgl. hierzu die Ergebnisse der Career-Concerns-Modelle von Amihud / Lev (1981), Holmström / Ricart I Costa (1986) und Hirshleifer / Thakor (1992), die zeigen, dass Manager aufgrund von Reputationsbedenken risikoreiche Investitionen meiden. Zu den folgenden Ausführungen siehe Fitch (2007), S. 15-18.

${ }^{21}$ Zur Ratingmethodik von CLOs siehe Moody's Investors Service (2009f), Standard \& Poor's (2002, 2009a) und Fitch (2008a). Da der Forderungspool einer Arbitrage CLO-Transaktion nicht statisch ist, sondern durch das aktive Management des CLO-Managers ständigen Veränderungen unterliegt, basiert die Bewertung der Ratingagenturen bei gemanagten CLOs auf dem hypothetischen Portfolio, welches sich ergibt, wenn der Manager die im Treuhandvertrag festgelegten Qualitäts-, Struktur- sowie Konzentrationsbeschränkungen gerade einhält. Vgl. hierzu Moody's Investors Service (2009f), S. 3, Standard \& Poor's (2002), S. 79-80, und Fitch (2008a), S. 13-15. Mit der Qualität des einer CLOTransaktion zugrunde liegenden Portfolios ist im Folgenden die Qualität des sich aus den Vorgaben des Treuhandvertrages ergebenden hypothetischen Referenzportfolios gemeint.

${ }^{22} \mathrm{Zu}$ den Sicherungsmaßnahmen von Verbriefungstransaktionen siehe z. B. Bär (2000), S. 207-227, Jobst (2002), S. 24-29, und Arlt (2009), S. 224-248. 
Enhancements ausgestattet ist. ${ }^{23}$ Da die Standardabweichung der Verlustverteilung eines Kreditportfolios mit sinkender Qualität der zugrunde liegenden Assets steigt, ist davon auszugehen, dass eine CLO-Tranche, die auf einem Referenzportfolio niedriger Qualität basiert, ceteris paribus eine höhere Downgrade-Wahrscheinlichkeit besitzt als eine vergleichbare Tranche, der ein Referenzportfolio von hoher Qualität zugrunde liegt. ${ }^{24}$ Hierzu können Keys u.a. (2009) und Güttler / Hommel / Reichert (2010) für US-amerikanische Mortgage Backed Securities einen positiven Zusammenhang zwischen dem FICO Score ${ }^{25}$ des zugrunde liegenden Asset Pools und dessen Performance nachweisen. Dies begründet

Hypothese 4: Die Wahrscheinlichkeit einer Ratingherabstufung sinkt mit steigender Qualität des Forderungspools.

Neben der Qualität des Asset Pools könnte auch die Größe der Equity Tranche (First Loss Piece, FLP) für die Performance einer CLO-Transaktion von Bedeutung sein. Ein möglicher Zusammenhang zwischen der Rating Performance und der Größe der Equity Tranche lässt sich anhand zweier Überlegungen begründen. Zum einen wird die Größe der Equity Tranche überwiegend durch die Qualität des zugrunde liegenden Portfolios bestimmt. ${ }^{26}$ Hierzu können Franke / Weber (2007) und Franke / Herrman / Weber (2007) einen inversen Zusammenhang zwischen der Größe der Equity Tranche und der Qualität des verbrieften Forderungsportfolios nachweisen. Sie führen dies auf mögliche Probleme asymmetrischer Informationsverteilung zwischen dem Originator und den Investoren zurück, die sich mit sinkender Portfolioqualität verstärken und zu deren Abschwächung daher der Einbehalt einer größeren Equity Tranche durch den Originator erforderlich ist. Dementsprechend ist von einem positiven Einfluss der Größe der Equity Tranche auf die Wahrscheinlichkeit einer Ratingherabstufung auszugehen. Andererseits ist zu berücksichtigen, dass die Equity Tranche aufgrund des Subordinationsprinzips den gerateten Tranchen als Verlustpuffer dient. Kommt es zu Verlusten im Referenzportfolio, werden diese zunächst von der Equity Tranche als Erstverlusttranche absorbiert. Erst wenn diese vollständig aufgezehrt ist, müssen die darüber liegenden Tranchen Verluste tragen. Folglich

${ }^{23}$ Der Attachment Point einer Tranche stellt die untere Grenze dar, ab welcher die Tranche von den Verlusten im Asset Pool betroffen wird. Der Detachment Point ist dagegen die obere Grenze, bei der die Tranche einen Totalverlust erleidet. Attachment und Detachment Point werden als kumulierte Verlustraten des Asset Pools angegeben. Vgl. Fender / Tarashev / Zhu (2008), S. 90.

${ }^{24}$ Zum Einfluss der Ausfallwahrscheinlichkeit und der Wiedergewinnungsquote der einer Transaktion zugrunde liegenden Kredite auf die Standardabweichung der Verlustverteilung siehe Scholz (2009), S. 29 und 42.

${ }^{25}$ Der FICO Score ist ein einfacher Kreditwürdigkeitsindex, der das relative Risiko eines Zahlungsausfalls oder eines erheblichen Zahlungsverzugs eines Schuldners ausdrückt. Der FICO Score berechnet sich auf Basis verschiedener Daten zur Kredithistorie eines Schuldners, beinhaltet jedoch keine Informationen über das Einkommen und das Vermögen eines Schuldners. Vgl. Fishelson-Holstine (2004), S. 5-7.

${ }^{26}$ Eine weitere Determinante der Höhe der Equity Tranche ist die Ausgestaltung des Subordinationsprinzips der CLO-Transaktion. Die Ergebnisse der Simulationen von Scholz (2009) zeigen, dass bei gegebenen Ratings für die vorrangigen Tranchen die Höhe einer möglichen Obergrenze des Reserve Accounts und die Ausgestaltung möglicher Coverage Tests die Größe der Equity Tranche beeinflussen. 
sinkt ceteris paribus die Ausfallwahrscheinlichkeit der gerateten Tranchen mit der Größe der Equity Tranche. Daher ist zu vermuten, dass bei gleicher Qualität des Referenzportfolios Transaktionen mit einer größeren Equity Tranche eine geringere Wahrscheinlichkeit einer Ratingherabstufung haben. Die Überlegungen zeigen, dass für die Erklärung eines möglichen Einflusses der Größe der Equity Tranche auf die Rating Performance zwei Ansätze mit gegensätzlichen Wirkungsrichtungen gefunden werden können. Stellt die Equity Tranche im Wesentlichen einen Proxy für die Qualität dar, ist von einem positiven Zusammenhang zwischen ihrer Größe und der Wahrscheinlichkeit einer Ratingherabstufung auszugehen. Dient die Equity Tranche hingegen vornehmlich als Verlustpuffer für die gerateten Tranchen, ist ein negativer Zusammenhang zu vermuten.

Hypothese 5: Die Wirkungsrichtung der Größe der Equity Tranche auf die Wahrscheinlichkeit einer Ratingherabstufung bestimmt sich in Abhängigkeit davon, ob die Equity Tranche vornehmlich als Proxy der Portfolioqualität oder als Verlustpuffer für die gerateten Tranchen dient.

Die Größe einer Transaktion könnte eine weitere Erklärungsdeterminante der Rating Performance darstellen, wenn unterstellt wird, dass die Granularität des einer Transaktion zugrunde liegenden Portfolios mit dem Transaktionsvolumen steigt. Da die Standardabweichung der Verlustverteilung eines Kreditportfolios mit zunehmender Diversifikation sinkt, sollten Transaktionen mit stärker diversifizierten Referenzportfolios eine geringere Downgrade-Wahrscheinlichkeit aufweisen als Transaktionen mit weniger diversifizierten Portfolios. ${ }^{27}$ Dies motiviert

Hypothese 6: Die Wahrscheinlichkeit einer Ratingherabstufung sinkt mit dem Transaktionsvolumen.

${ }^{27}$ Zum Einfluss der Diversifikation eines Kreditportfolios auf die Standardabweichung der Verlustverteilung siehe Scholz (2009), S. 37. 


\section{Empirische Auswertung europäischer Arbitrage CLO-Transaktionen}

\subsection{Datengrundlage}

In die vorliegende Untersuchung werden alle europäischen Arbitrage CLO-Transaktionen einbezogen, die im Zeitraum von September 1999 bis Juni 2008 begeben und bis Ende des Jahres 2008 noch nicht zurückgezahlt wurden. ${ }^{28}$ Von der Ratingagentur Fitch wurde ein Datensatz mit den in diesem Zeitraum emittierten Transaktionen sowie Informationen zu ihren Asset Managern zur Verfügung gestellt. Mittels verschiedener Informationsquellen wie Bloomberg, Moody's, Standard \& Poor's, Fitch sowie den Emissionsprospekten der Transaktionen wurde der Datensatz überprüft und um transaktions- sowie tranchenspezifische Charakteristika erweitert. Insgesamt umfasst der Datensatz 216 Transaktionen mit 1.992 Tranchen, die größtenteils durch Leveraged Loans gedeckt sind. ${ }^{29}$ Die Transaktionen werden von insgesamt 64 CLO-Managern verwaltet, von denen mehr als die Hälfte in den Jahren 2006 (21) und 2007 (15) in den europäischen CLO-Markt eingetreten sind. ${ }^{30}$

Um die Rating Performance der Transaktionen zu bestimmen, wurden für die CLOTranchen die Ratingherabstufungen von Moody's, Standard \& Poor's und Fitch im Zeitraum von 01.01.2009 bis 31.12.2009 zusammengestellt. Der Grund für die Begrenzung des Beobachtungszeitraums auf das Jahr 2009 ist, dass die Ratingagenturen im Zuge der jüngsten Finanzkrise Schwächen in ihren Modellen zur Beurteilung der Bonität von Verbriefungen erkannten, woraufhin sie ihre Methoden und Annahmen zu deren Bewertung überarbeiteten. Als Konsequenz wurden sämtliche von den Agenturen vergebene Ratings strukturierter Wertpapiere überprüft, was eine Vielzahl an Ratingherabstufungen nach sich zog. Für europäische CLO-Transaktionen begannen die Ratingagenturen in der zweiten Jahreshälfte 2008 (Fitch) bzw. in der ersten Jahreshälfte 2009 (Moody's und Standard \& Poor's), ihre Ratingmethoden anzupassen und die Wertpapiere neu zu bewerten. ${ }^{31}$ So ist auf dem europäischen Markt für Arbitrage CLOs im Jahr 2009 eine Downgrade-Rate von rund $70 \%$ zu beobachten, während diese in den Jahren von 2001 bis

\footnotetext{
${ }^{28}$ Da sämtliche in den Jahren 1999 und 2000 emittierten Transaktionen bis Ende des Jahres 2008 zurückgezahlt wurden, befinden sich letztlich nur Transaktionen der Jahre 2001 bis 2008 im Datensatz.

${ }^{29}$ Für zehn Transaktionen konnten keine Informationen zu den emittierten Tranchen gesammelt werden, weshalb sich die oben angegebene Tranchenanzahl auf nur insgesamt 206 Transaktionen bezieht.

${ }^{30}$ Zur Entwicklung der Anzahl der CLO-Manager am europäischen Markt siehe Fitch (2008b), S. 9-10, und Fitch (2010), S. 2-3.

${ }^{31}$ Nach der Aussage eines Vertreters der Ratingagentur Fitch wurden in 2008 lediglich die Ratings einer einzigen Transaktion von der Anpassung der Ratingmethodik betroffen. Für die Überarbeitung der Ratingmethodik von CLOs siehe Moody's Investors Service (2009a), Standard \& Poor's (2009a, 2009b) und Fitch (2008a). Hierbei wurden insbesondere die Annahmen hinsichtlich der Ausfallwahrscheinlichkeiten und Korrelationen der im Asset Pool befindlichen Forderungen angehoben.
} 
2008 deutlich unter 1\% lag. Hierbei ist jedoch anzumerken, dass die von den Agenturen in 2009 vorgenommenen Ratingherabstufungen nicht nur auf die geänderten Methoden und Annahmen zurückzuführen sind, sondern immer auch mit einer Verschlechterung der Qualität der zugrunde liegenden Forderungsportfolios begründet werden. ${ }^{32}$ Daher muss bei einer empirischen Analyse der Rating Performance von CLOs für die Änderung der Ratingmethodik kontrolliert werden oder eine separate Betrachtung der Ratingherabstufungen vor und nach der Überarbeitung erfolgen. Da jedoch bis Ende 2008 insgesamt nur acht Arbitrage CLO-Transaktionen eine Ratingherabstufung erfuhren, ist eine Analyse der Rating Performance vor Anpassung der Ratingmethodik aufgrund der geringen Anzahl an Beobachtungen nicht möglich. In der vorliegenden Studie wird daher ausschließlich der Zeitraum nach Überarbeitung der Ratingmethodik herangezogen. ${ }^{33}$ Hierbei konnten die Ratingänderungen im Jahr 2009 insgesamt nur für 205 Transaktionen ermittelt werden. ${ }^{34}$ Von den 1.989 Tranchen der 205 letztlich in die Untersuchung einbezogenen Transaktionen verfügen 1.708 Tranchen über ein Rating von mindestens einer der drei Ratingagenturen: 1.324 Tranchen sind von Moody's, 1.328 Tranchen von Standard \& Poor's und 233 Tranchen von Fitch bewertet. Die Anzahl nicht gerateter Tranchen, bei denen es sich überwiegend um Equity Tranchen handelt, beläuft sich auf 281 .

Die Performance der CLO-Transaktionen wird über die Dummyvariable DOWNGRADE beschrieben, die den Wert Eins annimmt, wenn mindestens eine Tranche der Transaktion in 2009 herabgestuft wurde, und andernfalls den Wert Null. ${ }^{35}$ Hierbei werden unter einer Ratingherabstufung nur tatsächliche Downgrades subsummiert. Veröffentlichungen von negativen Ausblicken (Outlook) oder Ankündigungen der Ratingagenturen, das Rating einer Tranche hinsichtlich einer möglichen Herabstufung zu überprüfen (Review bzw. Credit Watch) gelten nicht als Downgrade. ${ }^{36}$ Eine Betrachtung der in 2009 vorgenommenen Downgrades zeigt, dass 1.187 Tranchen der 1.708 gerateten Tranchen des Datensatzes

${ }^{32}$ Siehe hierzu z. B. die Ankündigungen von Moody's Investors Service (2009b, 2009c, 2009d, 2009e) in Bezug auf die im Zeitraum vom 17.03.2009 bis 20.03.2009 vorgenommenen Ratingherabstufungen sowie Fitch (2010), S. 4.

${ }^{33}$ Bei der Interpretation der in der empirischen Studie ermittelten Ergebnisse ist dementsprechend einschränkend zu berücksichtigen, dass sich der Untersuchungszeitraum auf eine Phase konjunkturellen Abschwungs bezieht. Vgl. hierzu auch die kritische Würdigung in Kapitel 4.6.

${ }^{34}$ Neben den zehn Transaktionen, für die keine Tranchendaten ermittelt werden konnte (siehe Fußnote 29), sind für eine weitere Transaktion keine Ratingdaten verfügbar.

${ }^{35}$ Eine von einer Ratingagentur vorgenommene Ratingherabstufung gilt nur dann als Downgrade, wenn das Tranchenrating dieser Agentur am Ende des Jahres 2009 niedriger ist als zu Beginn des Jahres. Demzufolge zählt eine Ratingherabstufung einer Agentur nicht als Downgrade, wenn diese einem Rating Upgrade der Agentur folgt und von ihrer Höhe, gemessen an der Anzahl der herabgestuften Ratingklassen, geringer ist als der vorherige Upgrade. Ein Überblick über die in der vorliegenden Studie herangezogenen Variablen und deren Bedeutung findet sich in Tabelle 6 des Anhangs.

${ }^{36}$ Der Ausblick einer Ratingagentur stellt deren Prognose bezüglich der mittelfristigen Entwicklung (12 bis 18 Monate) der Bonität eines Emittenten bzw. eines Finanzinstruments dar. Dieser kann entweder negativ, positiv oder stabil ausfallen. Stellt eine Ratingagentur einen Schuldner oder ein Finanzinstrument unter Beobachtung, so deutet dies auf eine mögliche (eher kurzfristig) kommende Ratingheraufstufung oder -herabstufung hin. Vgl. hierzu Micu / Remolona / Wooldridge (2004), S. 64. 
mindestens eine Ratingherabstufung erlitten, was einer Downgrade-Rate von knapp 70\% entspricht. Die herabgestuften Tranchen sind insgesamt 192 Transaktionen zugeordnet, so dass nur 13 Transaktionen des Datensatzes keinen Downgrade in 2009 erfuhren.

Der Einfluss möglicher durch die Vergütung induzierter Leistungs- und Risikoanreize von CLO-Managern auf die Rating Performance wird anhand der Subordinated Fee und der Incentive Fee, der variablen Bestandteile der Managervergütung, geprüft. ${ }^{37}$ Die Höhe der Subordinated Fee wird über die Variable SUBFEE gemessen. Sie ist definiert als jährlicher Prozentsatz des Nominalwerts des Asset Pools. Die in Tabelle 1 für die erklärenden Variablen angegebenen deskriptiven Statistiken zeigen, dass die Subordinated Fee im Mittel $0,40 \%$ p. a. beträgt, bei einem Wertebereich von $0,00 \%$ p. a. bis $0,75 \%$ p. a. Die von der Incentive Fee ausgehende Anreizwirkung wird über die Dummyvariable INCFEE geprüft, die die Existenz einer Incentive Fee im Management Agreement der Transaktion anzeigt. Von den 200 Transaktionen, für die die Managervergütung identifiziert werden konnte, wird in insgesamt 173 Transaktionen dem CLO-Manager eine Incentive Fee gewährt, wobei deren Ausgestaltungsformen variieren. Bei 134 Transaktionen ist die Incentive Fee als Anteil an den bei Überschreitung der Incentive Fee Threshold überschüssigen Cashflows definiert. Die Höhe dieses Anteils beträgt in 87\% der Fälle 20,00\%, bei einem Wertebereich von $8,30 \%$ bis $25,00 \%$. In 36 Transaktionen bestimmt sich die Höhe der Incentive Fee durch einen jährlichen Prozentsatz am Nominalwert des Asset Pools, der in der zugrunde gelegten Stichprobe zwischen 0,05\% p. a. und 0,38\% p. a. schwankt. ${ }^{38}$ Bei $99 \%$ der Transaktionen, die eine Incentive Fee für den Manager vorsehen, ist eine Threshold festgelegt. Diese ist in den überwiegenden Fällen konstant, jedoch finden sich auch Formen, bei denen sich die Threshold während der Laufzeit erhöht oder nach dem EURIBOR richtet.

Um den Einfluss der Erfahrung und der Größe eines CLO-Managers auf die Wahrscheinlichkeit einer Ratingherabstufung zu prüfen, werden zwei Maße herangezogen. Die Variable DEALS misst die Anzahl der bisher von einem Manager am europäischen Markt verwalteten CLO-Transaktionen, wobei das Closing Date der betrachteten Transaktion als Stichtag verwendet wird. Demnach werden sowohl die zum Emissionszeitpunkt noch ausstehenden Transaktionen als auch bereits zurückgezahlte Transaktionen des CLO-Managers berücksichtigt. Wie der Tabelle 1 zu entnehmen ist, beträgt zum Emissionszeitpunkt einer Transaktion die Anzahl der vom Manager verwalteten CLO-Transaktionen im Mittel 4,18 bei einem Median von 3,00. Mit einem minimalen Wert von 1,00 und einem maximalen

\footnotetext{
${ }^{37}$ Bei einigen Transaktionen, die hinsichtlich ihrer Anzahl jedoch eine untergeordnete Rolle spielen, setzt sich die performanceabhängige Vergütung des CLO-Managers aus mehr als zwei Komponenten zusammen. In diesen Fällen werden die Vergütungsbestandteile in Abhängigkeit von deren Priorität in Bezug auf die Verteilung der aus dem Pool eingehenden Zahlungen entweder der Subordinated Fee oder der Incentive Fee zugerechnet.

${ }^{38}$ Weitere drei Transaktionen sehen für den CLO-Manager eine individuell ausgestaltete Incentive Fee vor, die von den beiden typischen Formen abweicht.
} 
Tabelle 1: Deskriptive Statistiken der erklärenden Variablen. Für die metrisch skalierten Variablen wird die Anzahl der Beobachtungen (N), der Mittelwert, der Median, die Standardabweichung, das Minimum (Min) und das Maximum (Max) berichtet. Für die Dummyvariablen wird neben N, Min und Max der Anteil des beobachteten Merkmals angegeben.

\begin{tabular}{|c|c|c|c|c|c|c|}
\hline Variable & $\mathbf{N}$ & $\begin{array}{c}\text { Mittelwert/ } \\
\text { Anteil }\end{array}$ & Median & $\begin{array}{l}\text { Standard- } \\
\text { abweichung }\end{array}$ & Min & $\operatorname{Max}$ \\
\hline \multicolumn{7}{|l|}{ Managervergütung: } \\
\hline SUBFEE (in \%) & 200 & 0,40 & 0,45 & 0,14 & 0,00 & 0,75 \\
\hline INCFEE & 200 & $86,50 \%$ & & & 0,00 & 1,00 \\
\hline \multicolumn{7}{|c|}{ Managercharakteristika: } \\
\hline DEALS & 205 & 4,18 & 3,00 & 3,68 & 1,00 & 18,00 \\
\hline AUM (in Mio. EUR) & 205 & 1846,86 & 1133,25 & 1760,24 & 131,95 & 7631,30 \\
\hline LF SPEZIALIST & 205 & $44,39 \%$ & & & 0,00 & 1,00 \\
\hline LF BANK & 205 & $17,56 \%$ & & & 0,00 & 1,00 \\
\hline MULTI-ASSET & 205 & $22,93 \%$ & & & 0,00 & 1,00 \\
\hline HEDGE FOND & 205 & $8,78 \%$ & & & 0,00 & 1,00 \\
\hline PRIVATE EQUITY & 205 & $6,34 \%$ & & & 0,00 & 1,00 \\
\hline US & 205 & $30,73 \%$ & & & 0,00 & 1,00 \\
\hline \multicolumn{7}{|c|}{ Transaktionscharakteristika: } \\
\hline WARF & 196 & 779,26 & 738,67 & 324,94 & 48,63 & 2974,46 \\
\hline FLP (in \%) & 205 & 10,48 & 10,00 & 5,21 & 0,00 & 45,75 \\
\hline SIZE (in Mio. EUR) & 205 & 446,61 & 409,43 & 160,78 & 131,95 & 1140,00 \\
\hline \multicolumn{7}{|l|}{ Emissionsjahre: } \\
\hline$\overline{\mathrm{J} 2001}$ & 205 & $1,46 \%$ & & & 0,00 & 1,00 \\
\hline $\mathrm{J} 2002$ & 205 & $2,44 \%$ & & & 0,00 & 1,00 \\
\hline $\mathrm{J} 2003$ & 205 & $2,44 \%$ & & & 0,00 & 1,00 \\
\hline $\mathrm{J} 2004$ & 205 & $7,32 \%$ & & & 0,00 & 1,00 \\
\hline $\mathrm{J} 2005$ & 205 & $11,22 \%$ & & & 0,00 & 1,00 \\
\hline J2006 & 205 & $31,71 \%$ & & & 0,00 & 1,00 \\
\hline $\mathrm{J} 2007$ & 205 & $34,63 \%$ & & & 0,00 & 1,00 \\
\hline $\mathrm{J} 2008$ & 205 & $8,78 \%$ & & & 0,00 & 1,00 \\
\hline
\end{tabular}

Wert von 18,00 ist eine hohe Spannweite der Variable festzustellen, die mit dem starken Wachstum des europäischen CLO-Marktes bis 2007 zu begründen ist. Als zweites Maß für die Erfahrung und die Größe eines CLO-Managers wird die Variable AUM verwendet, die das von einem Manager verwaltete Vermögen in europäischen CLO-Transaktionen zum Emissionszeitpunkt der betrachteten Transaktion angibt. Das Volumen bereits zurückgezahlter Transaktionen des Managers findet hierbei keine Berücksichtigung. Tabelle 1 zeigt, dass ein Manager bei Closing einer Transaktion im Durchschnitt ein CLO-Vermögen in Europa von 1.847 Mio. EUR verwaltet. Mit einem Wertebereich von 132 Mio. EUR bis 7.631 Mio. EUR ist für die Variable AUM ebenfalls eine hohe Spannweite zu erkennen. In der vorliegenden Studie wird der natürliche Logarithmus der beiden Variablen, lnDEALS und lnAUM, herangezogen.

Des Weiteren lassen sich CLO-Manager nach ihrem Typ und dem Sitz ihres Unternehmens unterscheiden. In Bezug auf den Typ können die CLO-Manager des Datensatzes fünf Gruppen zugeordnet werden: Leverage Finance Spezialist (23), Leverage Finance Abteilung einer Bank (16), Multi-Asset Manager (14), Hedge Fond (7) und Private EquityGesellschaft (4). Hierbei werden $44 \%$ der im Datensatz befindlichen Transaktionen von Leverage Finance Spezialisten verwaltet, 18\% von Investmentbanken, 23\% von Multi- 
Asset Managern, 9\% von Hedge Fonds und 6\% von Private Equity-Unternehmen. In Bezug auf den Unternehmenssitz wird zwischen Europa (41) und USA (23) differenziert. 69\% der Transaktionen des Datensatzes werden von CLO-Managern mit Sitz in Europa, 31\% von CLO-Managern mit Sitz in den USA gemanagt. ${ }^{39}$

Zur Beschreibung der Qualität des einer Transaktion zugrunde liegenden Portfolios bieten sich verschiedene Maße an. ${ }^{40}$ Für die vorliegende Studie wird der von Schaber (2009) verwendete gewichtete durchschnittliche Ratingfaktor WARF (Weighted Average Rating Factor) einer Transaktion herangezogen, der sich aus den zum Emissionszeitpunkt vorliegenden Ratings der einzelnen Tranchen einer CLO-Transaktion errechnet. ${ }^{41}$ Hierzu werden die Tranchenratings in Ratingfaktoren transformiert, welche für die Ratingklassen die mit 10.000 multiplizierten ,idealisierten“ zehnjährigen Ausfallraten von Moody's angeben. ${ }^{42}$ Der WARF berechnet sich als gewichteter Durchschnitt der für die Tranchen einer CLOTransaktion ermittelten Ratingfaktoren, wobei die Gewichtung mit dem am Nominalwert gemessenen Anteil einer Tranche am gesamten Transaktionsvolumen erfolgt. ${ }^{43}$ Nicht gerateten Equity Tranchen wird in Analogie zu Schaber (2009) ein Ratingfaktor von 6.500 zugewiesen, der mit einem Rating von Caa2 korrespondiert. Transaktionen, bei denen eine oder mehrere vorrangige Tranchen (Senior oder Mezzanine Tranchen) über kein Rating einer der drei Agenturen verfügen, werden aus der Betrachtung ausgeschlossen, weshalb die Variable WARF nur für 196 Transaktionen des Datensatzes vorliegt. Verfügt eine Tranche über das Rating von mehr als einer Agentur, werden die entsprechenden Ratingfaktoren der Tranche gemittelt. ${ }^{44}$ Da die Ratingfaktoren nicht linear, sondern exponentiell mit der Ratingklasse ansteigen, haben Tranchen mit einem niedrigen Rating einen stärkeren Einfluss auf den durchschnittlichen Ratingfaktor als höher eingestufte Tranchen. Wie in Tabelle 1 berichtet ist, beträgt der WARF im Mittel 779. Dieser korrespondiert mit

\footnotetext{
${ }^{39}$ Die in der Studie für den Typ und den Unternehmenssitz eines CLO-Managers verwendeten Variablen sind in Tabelle 6 des Anhangs zu finden.

${ }^{40}$ Beispielsweise verwenden Franke / Weber (2007), Franke / Herrman / Weber (2007) und Franke / Weber (2009) in ihren empirischen Studien zur Gestaltung von CDO-Transaktionen zwei von Moody's publizierte Qualitätsmaße, die durchschnittliche Ausfallwahrscheinlichkeit des Forderungsportfolios (Weighted Average Default Probability) und den Moody's Diversity Score, der ein Maß für die Risikostreuung eines Portfolios darstellt. Cuchra (2005) und Cuchra / Jenkinson (2006) ziehen als Qualitätsmaß ein selbst konstruiertes Durchschnittsrating heran, das sich aus den Ratings der einzelnen Tranchen einer Transaktion errechnet. Keys u. a. (2009) und Güttler / Hommel / Reichert (2010) verwenden in ihren Performancestudien den FICO Score und die Beleihungsrate (Loan-to-Value Ratio) als spezifische Qualitätsmaße für Hypothekenkredite.

${ }^{41}$ Vgl. Schaber (2009), S. 149-150.

42 Die idealisierten Ausfallraten basieren auf den von Moody's ermittelten historischen Ausfallraten. Siehe Moody's Investors Service (2009f), S. 3-4. Die zu den Ratingklassen der Agenturen zugeordneten Ratingfaktoren sind in Moody's Investors Service (2009f), S. 33, sowie in Tabelle 7 des Anhangs zu finden.

${ }^{43}$ Hierbei ist anzumerken, dass Moody's zur Bewertung von CLOs den durchschnittlichen Ratingfaktor des Asset Pools heranzieht, der nicht wie der durchschnittliche Ratingfaktor einer Transaktion auf den Ratingfaktoren der Tranchen, sondern auf denen der einzelnen Vermögenswerte im Asset Pool basiert.

44 Tranchen mit Split Ratings kommt jedoch mit einem Anteil von 5\% nur eine untergeordnete Bedeutung zu. Lediglich 87 der 1.709 Tranchen verfügen über ein Split Rating.
} 
einer idealisierten zehnjährigen Ausfallwahrscheinlichkeit von 7,79\% bzw. einem Rating zwischen Baa3 und Ba1. Mit einer minimalen Ausprägung des durchschnittlichen Ratingfaktors von 49 (Aa3 bis A1) und einer maximalen Ausprägung von 2.974 (B2 bis B3) sind für die zugrunde gelegte Stichprobe große Qualitätsunterschiede zu konstatieren. In der vorliegenden Regressionsanalyse wird der natürliche Logarithmus des durchschnittlichen Ratingfaktors, lnWARF, verwendet.

Die Größe der Equity Tranche als mögliche Erklärungsdeterminante der Wahrscheinlichkeit einer Ratingherabstufung wird durch ihren am Nominalwert gemessenen Anteil am Transaktionsvolumen beschrieben. Als Equity Tranche gelten hierbei diejenigen Tranchen, die die geringste Priorität in Bezug auf die aus dem Asset Pool eingehenden Zahlungen besitzen und über kein Rating einer der drei Agenturen verfügen. ${ }^{45}$ Wie in Tabelle 1 an der die Größe der Equity Tranche angebenden Variable FLP zu erkennen ist, macht die Equity Tranche im Mittel 10,48\% am Transaktionsvolumen aus, wobei mit einem Wertebereich von $0,00 \%$ bis $45,75 \%$ für die Variable FLP eine hohe Spannweite festzustellen ist. Das Transaktionsvolumen als Proxy für die Granularität des Referenzportfolios wird schließlich über die Variable SIZE gemessen, die als Summe der Nominalwerte der Tranchen einer CLO-Transaktion definiert ist. ${ }^{46}$ Wie Tabelle 1 zeigt, beträgt das Transaktionsvolumen durchschnittlich 447 Mio. EUR. Für die Regressionsanalyse wird der natürliche Logarithmus des Transaktionsvolumens, lnSIZE, verwendet.

\subsection{Univariate Ergebnisse}

Als erster Schritt der Analyse werden die CLO-Transaktionen nach der Ausprägung der Variable DOWNGRADE in zwei Teilstichproben, Transaktionen mit und ohne Ratingherabstufung, unterteilt und die Charakteristika der beiden Stichproben verglichen. Die Ergebnisse sind in Tabelle 2 dargestellt. Wie zu erkennen ist, umfasst die Teilstichprobe der CLO-Transaktionen ohne Ratingherabstufung 13 Beobachtungen und die Teilstichprobe der CLO-Transaktionen mit einer oder mehreren Ratingherabstufungen 192 Beobachtungen. ${ }^{47}$ Der Vergleich der Charakteristika zeigt, dass hinsichtlich dreier Variablen signifikante Unterschiede zwischen den beiden Teilstichproben bestehen. Ein hochsignifi-

\footnotetext{
${ }^{45}$ Im Fall mehrerer subordinierter Tranchen ohne Rating wird die Summe der Anteile dieser Tranchen herangezogen. Verfügt die unterste Tranche dagegen über ein Rating, so gilt diese nicht als Equity Tranche und es wird für ihre Größe ein Wert von Null festgesetzt.

${ }^{46}$ Hierbei ist anzumerken, dass bei der Berechnung des Transaktionsvolumens die Nominalwerte der häufig im Rahmen von CLO-Transaktionen emittierten Combination Notes nicht berücksichtigt werden, da diese sich aus Teilen der „normalen“ Tranchen einer Transaktion zusammensetzen. Für eine Darstellung der Combination Notes siehe Schaber (2009), S. 87-88.

${ }^{47}$ Aufgrund fehlender Beobachtungen umfasst die Teilstichprobe der CLO-Transaktionen mit Ratingherabstufung bezüglich der Variablen SUBFEE und INCFEE lediglich 187 Beobachtungen und bezüglich der Variable WARF nur 183 Beobachtungen.
} 
Tabelle 2: Vergleich der Charakteristika von Arbitrage CLO-Transaktionen mit und ohne Ratingherabstufung. Für die metrisch skalierten Variablen werden pro Teilstichprobe die Anzahl der Beobachtungen $(\mathrm{N})$, der Mittelwert, die Standardabweichung und der p-Wert des t-Tests auf Gleichheit der Mittelwerte berichtet. Für die Dummyvariablen werden pro Teilstichprobe neben N der Anteil des beobachteten Merkmals und der p-Wert des $\chi^{2}$-Homogenitätstests auf Gleichheit der Verteilungen dokumentiert. Auf signifikante Unterschiede hinweisende p-Werte sind mit Asterisken gekennzeichnet, wobei ${ }^{* * *},{ }^{* *}$ und ${ }^{*}$ signifikant auf dem 1\%-, 5\%- bzw. 10\%-Niveau bedeutet (zweiseitige Tests).

\begin{tabular}{|c|c|c|c|c|c|c|c|}
\hline \multirow[b]{2}{*}{ Variable } & \multicolumn{3}{|c|}{ DOWNGRADE $=0$} & \multicolumn{3}{|c|}{ DOWNGRADE=1 } & \multirow[b]{2}{*}{ p-Wert } \\
\hline & $\mathbf{N}$ & $\begin{array}{c}\text { Mittelwert/ } \\
\text { Anteil }\end{array}$ & $\begin{array}{l}\text { Standard- } \\
\text { abweichung }\end{array}$ & $\mathbf{N}$ & $\begin{array}{c}\text { Mittelwert / } \\
\text { Anteil }\end{array}$ & $\begin{array}{l}\text { Standard- } \\
\text { abweichung }\end{array}$ & \\
\hline \multicolumn{8}{|l|}{ Managervergütung: } \\
\hline SUBFEE & 13 & 0,34 & 0,18 & 187 & 0,40 & 0,14 & 0,154 \\
\hline INCFEE & 13 & $46,15 \%$ & & 187 & $89,30 \%$ & & $0,000^{* * *}$ \\
\hline \multicolumn{8}{|c|}{ Managercharakteristika: } \\
\hline DEALS & 13 & 6,00 & 4,81 & 192 & 4,05 & 3,58 & $0,065^{*}$ \\
\hline AUM & 13 & 2883,21 & 2497,07 & 192 & 1776,69 & 1685,09 & $0,028^{* *}$ \\
\hline LF SPEZIALIST & 13 & $61,54 \%$ & & 192 & $43,23 \%$ & & 0,198 \\
\hline LF BANK & 13 & $23,08 \%$ & & 192 & $17,19 \%$ & & 0,589 \\
\hline MULTI-ASSET & 13 & $15,38 \%$ & & 192 & $23,44 \%$ & & 0,504 \\
\hline HEDGE FOND & 13 & $0,00 \%$ & & 192 & $9,38 \%$ & & 0,248 \\
\hline PRIVATE EQUITY & 13 & $0,00 \%$ & & 192 & $6,77 \%$ & & 0,332 \\
\hline US & 13 & $38,46 \%$ & & 192 & $30,21 \%$ & & 0,533 \\
\hline \multicolumn{8}{|c|}{ Transaktionscharakteristika: } \\
\hline WARF & 13 & 826,05 & 715,00 & 183 & 775,93 & 281,51 & 0,592 \\
\hline FLP & 13 & 11,29 & 11,28 & 192 & 10,43 & 4,58 & 0,565 \\
\hline SIZE & 13 & 510,91 & 213,53 & 192 & 442,25 & 156,34 & 0,137 \\
\hline
\end{tabular}

kanter Unterschied ergibt sich hinsichtlich der Verteilung der Dummyvariable INCFEE, die die Existenz einer Incentive Fee im Management Agreement einer CLO-Transaktion anzeigt. Während bei den Transaktionen ohne Downgrade nur 46,15\% eine Incentive Fee dem CLO-Manager gewähren, sind es bei den Transaktionen mit Downgrade 89,30\%. Des Weiteren zeigen sich Unterschiede in den Mittelwerten der die Erfahrung und die Größe eines CLO-Managers messenden Variablen DEALS und AUM. Die Variable DEALS, definiert als die Anzahl der bisher vom Asset Manager verwalteten europäischen CLOTransaktionen, beträgt für Transaktionen ohne Downgrade im Mittel 6,00, wohingegen sich für Transaktionen mit Downgrade ein Mittelwert von 4,05 ergibt. Ebenso ist eine höhere Erfahrung und Größe der CLO-Manager von nicht herabgestuften Transaktionen an der Variable AUM zu erkennen, die das von einem Manager zum Closing Date einer Transaktion verwaltete Vermögen in europäischen CLO-Transaktionen misst. Während die CLO-Manager der Transaktionen ohne Downgrade über ein verwaltetes Vermögen von durchschnittlich 2.883 Mio. EUR verfügen, sind es bei den CLO-Managern der Transaktionen mit Downgrade im Durchschnitt nur 1.777 Mio. EUR. Bezüglich der herangezogenen transaktionsspezifischen Charakteristika ergeben sich dagegen keine signifikanten Mittelwertunterschiede zwischen den Teilstichproben. 


\subsection{Multivariate Ergebnisse}

Die in Kapitel 3 abgeleiteten Hypothesen werden anhand mehrerer binärer Probitmodelle geprüft, die als abhängige Variable die Dummyvariable DOWNGRADE verwenden. ${ }^{48}$ Zunächst wird der Einfluss möglicher durch die Managervergütung hervorgerufener Anreizeffekte sowie der Einfluss der weiteren manager- und transaktionsspezifischen Charakteristika auf die Downgrade-Wahrscheinlichkeit einer Arbitrage CLO-Transaktion isoliert betrachtet, bevor dann deren Erklärungskraft in mehreren vollständigen Modellen gemeinsam untersucht wird. Es werden Dummyvariablen für die Emissionsjahre der Transaktionen zur Kontrolle für jahresspezifische Einflüsse hinzugezogen, wobei die Emissionsjahrgänge 2001 / 2002, 2003 / 2004, 2005 / 2006 und 2007 / 2008 jeweils zu einer Dummyvariablen zusammengefasst werden. ${ }^{49}$ Tabelle 3 zeigt die Ergebnisse der Probitregressionen, anhand derer der Einfluss der variablen Managervergütung, der Manager- und der Transaktionseigenschaften jeweils einzeln geprüft wird.

Im Modell I werden die Variable SUBFEE für die Höhe der Subordinated Fee und die die Existenz einer Incentive Fee anzeigende Dummyvariable INCFEE in die Regression einbezogen. Wie aus Tabelle $3 \mathrm{zu}$ erkennen ist, ist der Regressionskoeffizient für den INCFEE-Dummy positiv und hochsignifikant. Die Wahrscheinlichkeit einer Ratingherabstufung ist folglich höher für Transaktionen, bei denen dem CLO-Manager eine Incentive Fee gewährt wird. Dieser Befund weist darauf hin, dass durch die Incentive Fee Anreize für CLO-Manager geschaffen werden, eine risikoreiche Anlagestrategie für das zugrunde liegende Portfolio zu verfolgen. In Bezug auf den in Hypothese 2 formulierten Trade-Off zwischen den beiden mit der Incentive Fee verbundenen Anreizeffekten zeigt der positive Koeffizient von INCFEE, dass im Untersuchungszeitraum die Auswirkungen des Risk Shifting-Problems mögliche positive Leistungsanreizeffekte für CLO-Manager übersteigen. Hierbei ist davon auszugehen, dass sich eine risikoreiche Investitionspolitik insbesondere in Krisenzeiten, wie es im herangezogenen Untersuchungszeitraum der Fall ist, negativ

\footnotetext{
${ }^{48}$ Für eine ausführliche Darstellung von Probitmodellen siehe z. B. Greene (2000), S. 812-837, und Wooldridge (2009), S. 575-587.

${ }^{49}$ Der Grund für die Zusammenfassung der Jahrgänge besteht zum einen darin, dass für die Emissionsjahrgänge 2001 bis 2003 nur eine geringe Anzahl an Beobachtungen vorliegt. Siehe die deskriptiven Statistiken der Emissionsjahrgänge in Tabelle 1. Zum anderen wurden sämtliche in 2003 und 2005 emittierten Transaktionen des Datensatzes in 2009 herabgestuft. Daher müssen bei Einbezug von Jahresdummies die Dummyvariablen für 2003 und 2005 zusammen mit den in diesen Jahren emittierten Transaktionen aus der Regressionsschätzung ausgeschlossen werden, wodurch sich die Anzahl der Beobachtungen auf 177 Transaktionen reduzieren würde. Der Grund hierfür ist, dass die abhängige Variable DOWNGRADE für diese Emissionsjahre keine Schwankung aufweist, so dass bei Einbezug der in diesen Jahren emittierten Transaktionen eine Maximum-Likelihood-Schätzung nicht möglich wäre. Die im Folgenden dargestellten Regressionsschätzungen wurden ebenfalls für den reduzierten Datensatz bei Einbezug von Jahresdummies durchgeführt. Hierbei wurden die Ergebnisse der anhand des vollständigen Datensatzes vorgenommenen Schätzungen nicht beeinflusst, es ergaben sich lediglich geringe Unterschiede in den Signifikanzen.
} 
Tabelle 3: Determinanten der Rating Performance von Arbitrage CLO-Transaktionen für das Jahr 2009 Ergebnisse I. Es werden Schätzergebnisse binärer Probitregressionen zur Erklärung der Determinanten der Wahrscheinlichkeit einer Ratingherabstufung berichtet. Die abhängige Variable entspricht der Dummyvariablen DOWNGRADE, die die Ratingherabstufung einer oder mehrerer Tranchen einer Transaktion in 2009 anzeigt. Mit N wird die Anzahl der Beobachtungen gekennzeichnet. Standardfehler in Klammern sind robust für Heteroskedastizität. Signifikante Koeffizienten sind mit Asterisken gekennzeichnet, wobei ***, ** und * signifikant auf dem 1\%-, 5\%- bzw. 10\%-Niveau bedeutet (zweiseitige Tests).

\begin{tabular}{|c|c|c|c|c|c|}
\hline Variable & Modell I & Modell II & Modell III & Modell IV & Modell V \\
\hline SUBFEE & $\begin{array}{c}0,000 \\
(1,026)\end{array}$ & & & & \\
\hline INCFEE & $\begin{array}{l}1,118^{* * *} \\
(0,374)\end{array}$ & & & & \\
\hline $\operatorname{lnDEALS}$ & & $\begin{array}{c}-0,577^{* * *} \\
(0,189)\end{array}$ & & & \\
\hline $\ln \mathrm{AUM}$ & & & $\begin{array}{c}-0,488^{* *} \\
(0,190)\end{array}$ & & \\
\hline LF SPEZIALIST & & $\begin{array}{r}-0,537 \\
(0,365)\end{array}$ & $\begin{array}{r}-0,489 \\
(0,364)\end{array}$ & & \\
\hline LF BANK & & $\begin{array}{c}-1,056^{* *} \\
(0,497)\end{array}$ & $\begin{array}{c}-1,028^{* *} \\
(0,478)\end{array}$ & & \\
\hline US & & $\begin{array}{c}-0,818^{* * *} \\
(0,298)\end{array}$ & $\begin{array}{c}-0,766^{* *} \\
(0,303)\end{array}$ & & \\
\hline $\operatorname{lnWARF}$ & & & & $\begin{array}{c}0,085 \\
(0,328)\end{array}$ & \\
\hline FLP & & & & & $\begin{array}{r}-0,014 \\
(0,027)\end{array}$ \\
\hline $\operatorname{lnSIZE}$ & & & & $\begin{array}{r}-0,490 \\
(0,433)\end{array}$ & $\begin{array}{r}-0,493 \\
(0,435)\end{array}$ \\
\hline J0304 & $\begin{array}{c}1,007 \\
(0,804)\end{array}$ & $\begin{array}{c}1,294^{*} \\
(0,703)\end{array}$ & $\begin{array}{c}1,174^{*} \\
(0,707)\end{array}$ & $\begin{array}{c}0,847 \\
(0,697)\end{array}$ & $\begin{array}{c}0,886 \\
(0,699)\end{array}$ \\
\hline J0506 & $\begin{array}{l}1,429^{* *} \\
(0,673)\end{array}$ & $\begin{array}{l}1,841^{* * *} \\
(0,648)\end{array}$ & $\begin{array}{l}1,721^{* * *} \\
(0,648)\end{array}$ & $\begin{array}{l}1,266^{* *} \\
(0,577)\end{array}$ & $\begin{array}{l}1,339^{* *} \\
(0,583)\end{array}$ \\
\hline J0708 & $\begin{array}{c}0,794 \\
(0,618)\end{array}$ & $\begin{array}{l}1,402^{* *} \\
(0,604)\end{array}$ & $\begin{array}{l}1,275^{* *} \\
(0,594)\end{array}$ & $\begin{array}{c}0,640 \\
(0,528)\end{array}$ & $\begin{array}{c}0,730 \\
(0,536)\end{array}$ \\
\hline KONSTANTE & $\begin{array}{r}-0,276 \\
(0,656)\end{array}$ & $\begin{array}{l}1,620^{* * *} \\
(0,555)\end{array}$ & $\begin{array}{l}4,513^{* * *} \\
(1,347)\end{array}$ & $\begin{array}{c}3,116 \\
(3,004)\end{array}$ & $\begin{array}{c}3,796 \\
(2,693)\end{array}$ \\
\hline $\mathrm{N}$ & 200 & 205 & 205 & 196 & 205 \\
\hline Log-Likelihood & $-38,472$ & $-40,167$ & $-40,479$ & $-43,577$ & $-44,103$ \\
\hline McFaddens $\mathrm{R}^{2}$ & 0,200 & 0,171 & 0,164 & 0,089 & 0,089 \\
\hline p-Wert (Wald $\chi^{2}$ ) & 0,004 & 0,022 & 0,038 & 0,292 & 0,239 \\
\hline
\end{tabular}

auf die Performance von CLOs auswirkt und bestehende Leistungsanreizeffekte dominiert. Für die Variable SUBFEE kann dagegen kein Einfluss auf die Wahrscheinlichkeit einer Ratingherabstufung nachgewiesen werden. Ihr Koeffizient ist nicht signifikant und besitzt entgegen der Hypothese 1 ein positives Vorzeichen. Insgesamt weist das Modell I mit einem McFaddens $R^{2}$ von 0,20 einen mittleren Erklärungsgehalt auf.

In den Modellen II und III wird die Bedeutung der ausgewählten Managercharakteristika als Erklärungsdeterminanten der Downgrade-Wahrscheinlichkeit von CLO-Transaktionen geprüft. Neben den Variablen lnDEALS und lnAUM für die Erfahrung und die Größe eines CLO-Managers werden der US-Dummy für den US-amerikanischen Unternehmenssitz eines Managers und die den Managertyp anzeigenden Dummyvariablen LF SPEZIALIST und LF BANK in die Regression einbezogen. ${ }^{50}$ Hierbei wird aufgrund der Multikollinearität zwischen den Variablen lnDEALS und lnAUM von einer simultanen Betrachtung

\footnotetext{
${ }^{50}$ Aufgrund der geringen Anzahl an CLO-Managern, die den drei Managertypen Multi-Asset Manager, Hedge Fond und Private Equity-Gesellschaft angehören, werden diese zur Referenzgruppe zusammen-
} 
dieser Variablen abgesehen. ${ }^{51}$ In beiden Modellspezifikationen kann, wie in Hypothese 3 unterstellt, ein negativer Zusammenhang zwischen der Erfahrung bzw. der Größe eines Managers und der Wahrscheinlichkeit einer Ratingherabstufung festgestellt werden. Der Koeffizient von lnDEALS, definiert als die logarithmierte Anzahl der bisher von einem Manager verwalteten europäischen CLO-Transaktionen, deutet auf eine mit der Managererfahrung und -größe sinkende Downgrade-Wahrscheinlichkeit hin. Dieser Zusammenhang findet auch in der Variable lnAUM Bestätigung. Die Wahrscheinlichkeit einer Ratingherabstufung sinkt ebenfalls mit dem logarithmierten Vermögen in europäischen CLO-Transaktionen, das der Asset Manager zum Closing Date einer Transaktion verwaltet. Bezüglich der Bedeutung des Managertyps für die Downgrade-Wahrscheinlichkeit einer Transaktion zeigt sich in beiden Modellen für den LF BANK-Dummy ein signifikant negativer Koeffizient. Dieser gibt an, dass Transaktionen, die von einer Investmentbank (Leverage Finance Abteilung) gemanagt werden, mit einer geringeren Wahrscheinlichkeit in 2009 herabgestuft wurden als Transaktionen mit einem Multi-Asset Manager, einem Hedge Fond oder einer Private Equity-Gesellschaft als CLO-Manager. Der Koeffizient der Dummyvariable LF SPEZIALIST ist dagegen nicht signifikant, weist jedoch gleichermaßen ein negatives Vorzeichen auf, welches eine im Vergleich zur Referenzgruppe geringere Downgrade-Wahrscheinlichkeit von CLO-Transaktionen andeutet, die von einem Leverage Finance Spezialisten verwaltet werden. Für den US-Dummy kann ebenfalls ein Zusammenhang mit der Wahrscheinlichkeit einer Ratingherabstufung festgestellt werden. Der negative Koeffizient zeigt an, dass Transaktionen US-amerikanischer CLOManager eine im Durchschnitt geringere Downgrade-Wahrscheinlichkeit im Jahr 2009 aufweisen als Transaktionen europäischer Manager. Dieses Ergebnis lässt vermuten, dass US-amerikanische CLO-Manager aufgrund ihrer Managementtätigkeiten auf dem amerikanischen CLO-Markt, welche in den Variablen DEALS und AUM aufgrund der Beschränkung auf europäische Transaktionen nicht berücksichtigt sind, eine höhere Erfahrung im Management von CLO-Transaktionen besitzen als ihre europäischen Wettbewerber. Der Erklärungsgehalt der Modelle II und III ist jedoch mit einem McFaddens $\mathrm{R}^{2}$ von 0,17 und 0,16 eher gering.

In den Modellen IV und V wird schließlich der Einfluss der als mögliche Erklärungsdeterminanten identifizierten Transaktionseigenschaften auf die Wahrscheinlichkeit einer Ratingherabstufung untersucht. Im Modell IV werden die Variablen lnWARF und lnSIZE für die Qualität und die Größe einer CLO-Transaktion auf den DOWNGRADE-Dummy regressiert, im Modell V wird neben lnSIZE die die Größe der Equity Tranche messende Variable FLP in die Regression einbezogen. Aufgrund der Multikollinearität zwischen den

gefasst. Der Einfluss der beiden Managertypen Leverage Finance Spezialist und Leverage Finance Abteilung einer Bank wird somit jeweils im Vergleich zu dieser Referenzgruppe geprüft.

${ }^{51}$ Der Bravais-Pearson-Korrelationskoeffizient der Variablen beträgt 0,97. Die Korrelationsmatrix der erklärenden Variablen ist in Tabelle 8 des Anhangs angegeben. 
Variablen lnWARF und FLP bleibt deren gleichzeitige Aufnahme in die Regression aus. ${ }^{52}$ Die hohe Korrelation zwischen den beiden Variablen ist darauf zurückzuführen, dass bei der Berechnung des durchschnittlichen Ratingfaktors einer Transaktion der Equity Tranche der höchste Ratingfaktor von 6.500 zugewiesen wird und somit ihre Größe als deren Gewichtungsfaktor die Höhe des WARF erheblich beeinflusst. Die Schätzergebnisse der Modelle IV und V zeigen ein überraschendes Ergebnis: Die die Transaktionseigenschaften beschreibenden Variablen lnWARF, lnSIZE und FLP haben im Untersuchungszeitraum keinen signifikanten Einfluss auf die Rating Performance der Transaktionen. Die Vorzeichen der Koeffizienten dieser Variablen sind jedoch mit den in den Hypothesen 4 bis 6 unterstellten Zusammenhängen vereinbar. Die Hypothese 4 besagt, dass die Wahrscheinlichkeit einer Ratingherabstufung mit steigender Qualität einer CLO-Transaktion sinkt. Da die den durchschnittlichen Ratingfaktor messende Variable lnWARF einen umso höheren Wert annimmt, desto geringer die Qualität einer CLO-Transaktion ist, deutet der positive Koeffizient von lnWARF in Modell IV entsprechend der Hypothese 4 einen negativen Zusammenhang zwischen der Qualität und der Downgrade-Wahrscheinlichkeit einer CLO-Transaktion an. Der Koeffizient von lnSIZE besitzt in beiden Modellen ein negatives Vorzeichen, das in Übereinstimmung mit der Hypothese 6 eine mit der Größe einer Transaktion sinkende Downgrade-Wahrscheinlichkeit vermuten lässt. Der Koeffizient bleibt nahezu unverändert, wenn anstelle der Variable lnWARF die Variable FLP für die Größe der Equity Tranche in die Regression einbezogen wird. Gemäß der Hypothese 5 hängt die Richtung des Zusammenhangs zwischen der Größe der Equity Tranche und der Wahrscheinlichkeit einer Ratingherabstufung davon ab, ob die Equity Tranche überwiegend ein Proxy für die Qualität des Referenzportfolios darstellt oder den vorrangigen Tranchen als Verlustpuffer dient. Der negative Koeffizient von FLP deutet die Funktion der Equity Tranche als Verlustpuffer an. Demnach sinkt die Wahrscheinlichkeit einer Ratingherabstufung mit der Größe der Equity Tranche, da mit zunehmender Größe die Höhe an Verlusten steigt, die von der Equity Tranche getragen werden. Jedoch ist der Koeffizient von FLP nicht signifikant und das Modell V weist wie auch das Modell IV mit 0,09 einen sehr geringen Erklärungsgehalt auf.

Die Ergebnisse der Probitregressionen, in denen die möglichen Erklärungsdeterminanten gemeinsam auf den DOWNGRADE-Dummy regressiert werden, sind in Tabelle 4 dargestellt. Die Vorzeichen der Regressionskoeffizienten bleiben bis auf das des Koeffizienten der Variable SUBFEE in allen Modellspezifikationen gegenüber denen in Tabelle 3 unverändert. Bezüglich der Signifikanzen zeigen sich nur geringe Unterschiede. Der Koeffizient für die Höhe der Subordinated Fee bleibt weiterhin insignifikant, jedoch weist er nun in allen Modellen entsprechend der Hypothese 1 ein negatives Vorzeichen auf, das eine mit der

\footnotetext{
$\overline{52}$ Der Bravais-Pearson-Korrelationskoeffizient der Variablen beträgt 0,85. Siehe hierzu die Korrelations-
} matrix der erklärenden Variablen in Tabelle 8 des Anhangs. 
Tabelle 4: Determinanten der Rating Performance von Arbitrage CLO-Transaktionen für das Jahr 2009 Ergebnisse II. Es werden Schätzergebnisse binärer Probitregressionen zur Erklärung der Determinanten der Wahrscheinlichkeit einer Ratingherabstufung berichtet. Die abhängige Variable entspricht der Dummyvariablen DOWNGRADE, die die Ratingherabstufung einer oder mehrerer Tranchen einer Transaktion in 2009 anzeigt. Mit N wird die Anzahl der Beobachtungen gekennzeichnet. Standardfehler in Klammern sind robust für Heteroskedastizität. Signifikante Koeffizienten sind mit Asterisken gekennzeichnet, wobei $* * *, * *$ und $*$ signifikant auf dem 1\%-, 5\%- bzw. 10\%-Niveau bedeutet (zweiseitige Tests).

\begin{tabular}{|c|c|c|c|c|}
\hline Variable & Modell I & Modell II & Modell III & Modell IV \\
\hline SUBFEE & $\begin{array}{c}-0,450 \\
(0,946)\end{array}$ & $\begin{array}{c}-0,322 \\
(0,923)\end{array}$ & $\begin{array}{r}-0,633 \\
(0,915)\end{array}$ & $\begin{array}{c}-0,480 \\
(0,894)\end{array}$ \\
\hline INCFEE & $\begin{array}{l}1,154^{* * *} \\
(0,404)\end{array}$ & $\begin{array}{l}1,098^{* * *} \\
(0,396)\end{array}$ & $\begin{array}{l}1,077^{* * *} \\
(0,378)\end{array}$ & $\begin{array}{l}1,025^{* * *} \\
(0,370)\end{array}$ \\
\hline $\operatorname{lnDEALS}$ & $\begin{array}{c}-0,562^{* * *} \\
(0,195)\end{array}$ & & $\begin{array}{c}-0,557^{* * * *} \\
(0,185)\end{array}$ & \\
\hline $\ln \mathrm{AUM}$ & & $\begin{array}{c}-0,422^{* *} \\
(0,180)\end{array}$ & & $\begin{array}{c}-0,413^{* *} \\
(0,173)\end{array}$ \\
\hline LF SPEZIALIST & $\begin{array}{r}-0,379 \\
(0,387)\end{array}$ & $\begin{array}{r}-0,366 \\
(0,376)\end{array}$ & $\begin{array}{r}-0,466 \\
(0,381)\end{array}$ & $\begin{array}{r}-0,459 \\
(0,368)\end{array}$ \\
\hline LF BANK & $\begin{array}{c}-1,045^{* *} \\
(0,488)\end{array}$ & $\begin{array}{c}-0,988^{* *} \\
(0,480)\end{array}$ & $\begin{array}{c}-1,143^{* *} \\
(0,480)\end{array}$ & $\begin{array}{c}-1,073^{* *} \\
(0,470)\end{array}$ \\
\hline US & $\begin{array}{c}-0,888^{* *} \\
(0,351)\end{array}$ & $\begin{array}{c}-0,776^{* *} \\
(0,332)\end{array}$ & $\begin{array}{c}-0,805^{* *} \\
(0,341)\end{array}$ & $\begin{array}{c}-0,699 * * \\
(0,327)\end{array}$ \\
\hline $\operatorname{lnWARF}$ & $\begin{array}{c}0,051 \\
(0,255)\end{array}$ & $\begin{array}{c}0,069 \\
(0,252)\end{array}$ & & \\
\hline FLP & & & $\begin{array}{r}-0,012 \\
(0,025)\end{array}$ & $\begin{array}{c}-0,011 \\
(0,025)\end{array}$ \\
\hline $\operatorname{lnSIZE}$ & $\begin{array}{c}-0,425 \\
(0,467)\end{array}$ & $\begin{array}{r}-0,302 \\
(0,475)\end{array}$ & $\begin{array}{c}-0,445 \\
(0,491)\end{array}$ & $\begin{array}{c}-0,333 \\
(0,499)\end{array}$ \\
\hline J0304 & $\begin{array}{c}1,352 \\
(0,832)\end{array}$ & $\begin{array}{c}1,253 \\
(0,842)\end{array}$ & $\begin{array}{c}1,372^{*} \\
(0,829)\end{array}$ & $\begin{array}{c}1,271 \\
(0,838)\end{array}$ \\
\hline J0506 & $\begin{array}{l}1,943^{* *} \\
(0,764)\end{array}$ & $\begin{array}{l}1,798^{* *} \\
(0,755)\end{array}$ & $\begin{array}{l}2,049^{* * *} \\
(0,768)\end{array}$ & $\begin{array}{l}1,894^{* *} \\
(0,759)\end{array}$ \\
\hline J0708 & $\begin{array}{l}1,632^{* *} \\
(0,727)\end{array}$ & $\begin{array}{l}1,436^{* *} \\
(0,712)\end{array}$ & $\begin{array}{l}1,705^{* *} \\
(0,715)\end{array}$ & $\begin{array}{l}1,508^{* *} \\
(0,704)\end{array}$ \\
\hline KONSTANTE & $\begin{array}{c}2,896 \\
(3,436)\end{array}$ & $\begin{array}{c}4,478 \\
(3,552)\end{array}$ & $\begin{array}{c}3,608 \\
(3,084)\end{array}$ & $\begin{array}{c}5,295^{*} \\
(3,169)\end{array}$ \\
\hline $\mathrm{N}$ & 191 & 191 & 200 & 200 \\
\hline Log-Likelihood & $-33,724$ & $-34,296$ & $-34,339$ & $-34,953$ \\
\hline McFaddens $\mathrm{R}^{2}$ & 0,290 & 0,278 & 0,286 & 0,273 \\
\hline p-Wert (Wald $\chi^{2}$ ) & 0,000 & 0,001 & 0,001 & 0,001 \\
\hline
\end{tabular}

Höhe der Subordinated Fee sinkende Downgrade-Wahrscheinlichkeit vermuten lässt. Aufgrund der Insignifikanz des Koeffizienten kann die Hypothese 1, die aufgrund der mit der Subordinated Fee verbundenen positiven Leistungsanreizeffekte einen negativen Zusammenhang zwischen der Höhe der Subordinated Fee und der Rating Performance unterstellt, nicht bestätigt werden. In Bezug auf die Incentive Fee zeigt sich, dass der Koeffizient des INCFEE-Dummies weiterhin positiv und hochsignifikant ist. Hinsichtlich der Hypothese 2 kann also festgehalten werden, dass die Existenz einer Incentive Fee die Wahrscheinlichkeit einer Ratingherabstufung erhöht. Der Befund von Scholz (2009), dass die Incentive Fee Risikoanreize für CLO-Manager setzt, wird somit bestätigt. Allerdings kann das Ergebnis von Garrison (2005), dass die Auswirkungen des aus der Incentive Fee resultierenden Leistungsanreizeffekts die des Risikoanreizeffekts übersteigen, für den betrachteten Untersuchungszeitraum nicht nachgewiesen werden. Die Koeffizienten der Variablen lnDEALS und lnAUM für die Erfahrung und die Größe eines Managers zeigen unverändert einen negativen Zusammenhang mit der Wahrscheinlichkeit einer Ratingherabstufung an. Die Hypo- 
these 3, nach der die Wahrscheinlichkeit einer Ratingherabstufung mit der Erfahrung und der Größe eines CLO-Managers sinkt, wird somit sowohl für lnDEALS als auch für lnAUM bestätigt. In Bezug auf den Typ und den Unternehmenssitz eines CLO-Managers bleiben die Ergebnisse der Tabelle 3 ebenfalls bestehen. Die Dummyvariablen LF BANK und US verfügen weiterhin über signifikant negative Koeffizienten, wohingegen der negative Koeffizient der Dummyvariable LF SPEZIALIST keine Signifikanz besitzt. So kann festgehalten werden, dass im Untersuchungszeitraum Transaktionen, die von einer Investmentbank verwaltet werden, im Durchschnitt eine geringere Downgrade-Wahrscheinlichkeit aufweisen als die von Multi-Asset Managern, Hedge Fonds und Private Equity-Gesellschaften. Des Weiteren zeigen Transaktionen US-amerikanischer CLO-Manager eine bessere Rating Performance als Transaktionen europäischer CLO-Manager. Für die transaktionsspezifischen Merkmale ist aus Tabelle $4 \mathrm{zu}$ erkennen, dass die Qualität des Referenzportfolios, das Transaktionsvolumen und die Größe der Equity Tranche auch in den vollständigen Modellen keinen Einfluss auf die Downgrade-Wahrscheinlichkeit einer Transaktion haben. Zwar stimmen die Vorzeichen der Koeffizienten von lnWARF, lnSIZE und FLP wie zuvor mit den in den Hypothesen 4 bis 6 unterstellten Zusammenhängen überein, jedoch können die Hypothesen aufgrund der Insignifikanz der Koeffizienten in der vorliegenden Untersuchung nicht bestätigt werden. Schließlich ist in Bezug auf die Emissionsjahre noch anzumerken, dass die in 2005 und 2006 sowie die in 2007 und 2008 (1. Jahreshälfte) emittierten CLOTransaktionen im Untersuchungszeitraum eine höhere Downgrade-Wahrscheinlichkeit zeigen als Transaktionen aus den Jahren 2001 und 2002. Der Erklärungsgehalt der Modelle weist mit Werten zwischen 0,27 und 0,29 ein deutlich verbessertes Niveau im Vergleich zu den in Tabelle 3 berichteten Werten auf.

\subsection{Marginale Effekte der erklärenden Variablen}

Während bisher ausschließlich die Vorzeichen der geschätzten Regressionskoeffizienten analysiert wurden, steht nun die Höhe des Einflusses der erklärenden Variablen auf die Wahrscheinlichkeit einer Ratingherabstufung im Mittelpunkt der Betrachtung. Da Probitmodelle einen nicht-linearen Zusammenhang zwischen der Eintrittswahrscheinlichkeit der abhängigen Variable und den erklärenden Variablen unterstellen, ist der Einfluss der erklärenden Variablen nicht konstant, sondern hängt vom Niveau sämtlicher im Modell herangezogener Variablen ab. Daher werden zur Bestimmung der Höhe des Einflusses die marginalen Effekte der erklärenden Variablen berechnet. Diese geben den Effekt einer marginalen Veränderung einer Variablen auf die Downgrade-Wahrscheinlichkeit an, wobei alle anderen Variablen auf ihren Mittelwert gesetzt werden. Tabelle 5 zeigt die marginalen Effekte für die in Tabelle 4 berichteten Modellspezifikationen. Für die einbezogenen Dummyvariablen werden aufgrund ihrer dichotomen Natur die Effekte einer 
Tabelle 5: Marginale Effekte der erklärenden Variablen. Es werden die marginalen Effekte für die Modellspezifikationen der Tabelle 4 zur Erklärung der Determinanten der Rating Performance von Arbitrage CLO-Transaktionen für das Jahr 2009 berichtet. Die abhängige Variable entspricht der Dummyvariablen DOWNGRADE, die die Ratingherabstufung einer oder mehrerer Tranchen einer Transaktion in 2009 anzeigt. Für die einbezogenen Dummyvariablen werden die Effekte einer diskreten Änderung von Null auf Eins angegeben. Zur Berechnung der marginalen und der diskreten Effekte werden die erklärenden Variablen auf ihre Mittelwerte gesetzt. In den Modellen I und II werden 191 Transaktionen, in den Modellen III und IV 200 Transaktionen einbezogen. Standardfehler in Klammern sind robust für Heteroskedastizität. Signifikante Koeffizienten sind mit Asterisken gekennzeichnet, wobei $* * *, * *$ und $*$ signifikant auf dem 1\%-, 5\%-bzw. 10\%-Niveau bedeutet (zweiseitige Tests).

\begin{tabular}{|c|c|c|c|c|}
\hline Variable & Modell I & Modell II & Modell III & Modell IV \\
\hline SUBFEE & $\begin{array}{r}-0,027 \\
(0,055)\end{array}$ & $\begin{array}{c}-0,021 \\
(0,059)\end{array}$ & $\begin{array}{c}-0,035 \\
(0,049)\end{array}$ & $\begin{array}{c}-0,029 \\
(0,053)\end{array}$ \\
\hline INCFEE & $\begin{array}{c}0,154^{*} \\
(0,087)\end{array}$ & $\begin{array}{c}0,150 * \\
(0,086)\end{array}$ & $\begin{array}{c}0,130 * \\
(0,071)\end{array}$ & $\begin{array}{c}0,128^{*} \\
(0,070)\end{array}$ \\
\hline $\operatorname{lnDEALS}$ & $\begin{array}{c}-0,033^{* *} \\
(0,015)\end{array}$ & & $\begin{array}{c}-0,031^{* *} \\
(0,015)\end{array}$ & \\
\hline $\ln A U M$ & & $\begin{array}{c}-0,027^{* *} \\
(0,014)\end{array}$ & & $\begin{array}{c}-0,025^{*} \\
(0,013)\end{array}$ \\
\hline LF SPEZIALIST & $\begin{array}{c}-0,024 \\
(0,025)\end{array}$ & $\begin{array}{r}-0,025 \\
(0,027)\end{array}$ & $\begin{array}{c}-0,028 \\
(0,024)\end{array}$ & $\begin{array}{c}-0,030 \\
(0,025)\end{array}$ \\
\hline LF BANK & $\begin{array}{c}-0,123 \\
(0,102)\end{array}$ & $\begin{array}{c}-0,120 \\
(0,100)\end{array}$ & $\begin{array}{c}-0,135 \\
(0,102)\end{array}$ & $\begin{array}{c}-0,131 \\
(0,099)\end{array}$ \\
\hline US & $\begin{array}{r}-0,077^{*} \\
(0,040)\end{array}$ & $\begin{array}{r}-0,069^{*} \\
(0,038)\end{array}$ & $\begin{array}{r}-0,063^{*} \\
(0,036)\end{array}$ & $\begin{array}{c}-0,057^{*} \\
(0,034)\end{array}$ \\
\hline $\operatorname{lnWARF}$ & $\begin{array}{c}0,003 \\
(0,015)\end{array}$ & $\begin{array}{c}0,004 \\
(0,016)\end{array}$ & & \\
\hline FLP & & & $\begin{array}{c}-0,001 \\
(0,001)\end{array}$ & $\begin{array}{c}-0,001 \\
(0,002)\end{array}$ \\
\hline $\operatorname{lnSIZE}$ & $\begin{array}{c}-0,025 \\
(0,026)\end{array}$ & $\begin{array}{c}-0,020 \\
(0,029)\end{array}$ & $\begin{array}{c}-0,036 \\
(0,033)\end{array}$ & $\begin{array}{c}-0,020 \\
(0,028)\end{array}$ \\
\hline Jahresdummies & $\mathrm{Ja}$ & $\mathrm{Ja}$ & $\mathrm{Ja}$ & $\mathrm{Ja}$ \\
\hline
\end{tabular}

diskreten Veränderung von Null auf Eins angegeben. Wie aus Tabelle 5 zu erkennen ist, weisen die Modelle konsistente Schätzergebnisse auf. Bei durchweg gleichen Vorzeichen zeigen sich hinsichtlich der Höhe der marginalen und der diskreten Effekte nur leichte Unterschiede. Im Folgenden werden die geschätzten marginalen und diskreten Effekte anhand des Modells I erläutert, da dieses den höchsten Erklärungsgehalt besitzt. ${ }^{53}$ Es wird deutlich, dass die Wahrscheinlichkeit einer Ratingherabstufung zu einem Großteil von der Incentive Fee beeinflusst wird. Für Transaktionen, bei denen der CLO-Manager eine Incentive Fee erhält, ist die Wahrscheinlichkeit einer Ratingherabstufung im Vergleich zu Transaktionen ohne Incentive Fee um 15 Prozentpunkte höher. Andererseits sinkt die Wahrscheinlichkeit einer Ratingherabstufung um 3 Prozentpunkte, wenn sich die Anzahl der vom CLO-Manager verwalteten Transaktionen um 1\% erhöht. Für die ebenfalls die Erfahrung und die Größe eines CLO-Managers messende Variable lnAUM zeigen die in Modell II und IV geschätzten marginalen Effekte, dass die Downgrade-Wahrscheinlichkeit einer Transaktion sich um 3 Prozentpunkte verringert, wenn das zum Emissionszeitpunkt verwaltete CLO-Vermögen des Managers um 1\% steigt. Hinsichtlich des Unternehmenssitzes zeigt sich, dass im Untersuchungszeitraum Transaktionen US-amerikanischer CLOManager eine um 8 Prozentpunkte geringere Downgrade-Wahrscheinlichkeit aufweisen als

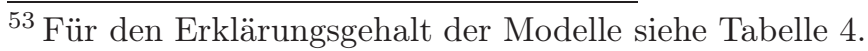


die europäischer CLO-Manager. Als signifikante Einflussgröße der Rating Performance erwies sich in der vorangegangenen Analyse zudem die den Typ anzeigende Dummyvariablen LF BANK. Ihr diskreter Effekt gibt an, dass die Downgrade-Wahrscheinlichkeit von CLO-Transaktionen, die von einer Investmentbank gemanagt werden, um 12 Prozentpunkte geringer ist als die von Transaktionen, bei denen ein Multi-Asset Manager, ein Hedge Fond oder eine Private Equity-Gesellschaft als CLO-Manager fungiert. Allerdings erweist sich der diskrete Effekt des LF BANK-Dummies als nicht signifikant.

\subsection{Klassifikationsfähigkeit des Probitmodells}

Zur Beurteilung der Modellgüte wird nun die Klassifikationsfähigkeit des Probitmodells analysiert. Hierzu wird die empirisch beobachtete Gruppenzuteilung, d. h. die Ausprägungen der abhängigen Variable DOWNGRADE, mit der über die Regressionsschätzung vorhergesagten Zuordnung verglichen. Die Zuordnung der Beobachtungen erfolgt über die ermittelten Downgrade-Wahrscheinlichkeiten, die einem zuvor festgelegten Trennwert (Cut Value) gegenübergestellt werden. Beobachtungen mit einer Downgrade-Wahrscheinlichkeit größer als der Trennwert werden der Gruppe der Transaktionen mit Ratingherabstufung zugerechnet, andernfalls der Gruppe der Transaktionen ohne Ratingherabstufung. Der Trennwert beeinflusst folglich die Gruppenzuteilung und damit die Klassifikationsfähigkeit des Modells. Der Einfluss des Trennwerts auf die Klassifikationsfähigkeit wird anhand der in Abbildung 1 dargestellten „Receiver Operating Characteristics (ROC)"-Kurve graphisch veranschaulicht. ${ }^{54}$ Die Abbildung zeigt die ROC-Kurve für das Modell I aus Tabelle 4, mit welchem die Klassifikationsfähigkeit des Regressionsansatzes im Folgenden beurteilt wird.

Die ROC-Kurve stellt die Sensitivität (Richtigpositiv-Rate) eines Modells in Abhängigkeit von dessen Spezifität für verschiedene Trennwerte dar. Die auf der Ordinate abgetragene Sensitivität entspricht hierbei dem Anteil der korrekt klassifizierten Transaktionen mit Ratingherabstufung an der Gesamtzahl der Transaktionen mit Ratingherabstufung. Auf der Abzisse befindet sich die Falschpositiv-Rate, die dem Ausdruck (1-Spezifität) entspricht und den Anteil der falsch klassifizierten Transaktionen ohne Ratingherabstufung an der Gesamtzahl der Transaktionen ohne Ratingherabstufung darstellt. ${ }^{55}$ Da von den insgesamt 205 in die Regression einbezogenen Beobachtungen nur 13 Transaktionen in 2009 nicht herabgestuft wurden, ist vor allem die Spezifität des Modells von Bedeutung, d.h. die Fähigkeit des Modells, die Transaktionen ohne Ratingherabstufung korrekt zu identifizieren. Die Diagonale in Abbildung 1 kennzeichnet die Klassifikationsergebnisse,

\footnotetext{
${ }^{54}$ Zur ROC-Analyse vgl. Bamber (1975), Hanley / McNeil (1982) und DeLong / DeLong / Clarke-Pearson (1988).

${ }^{55}$ Dementsprechend ist die Spezifität definiert als der Anteil der korrekt klassifizierten Transaktionen ohne Ratingherabstufung an der Gesamtanzahl der Transaktionen ohne Ratingherabstufung.
} 
Abbildung 1: ROC-Kurve des Probitmodells. Es werden die Schätzergebnisse der Modellspezifikation I der Tabelle 4 verwendet. Die ROC-Kurve stellt den Zusammenhang zwischen der Sensitivität und der Falschpositiv-Rate (1-Spezifität) in Abhängigkeit des gewählten Trennwerts dar.

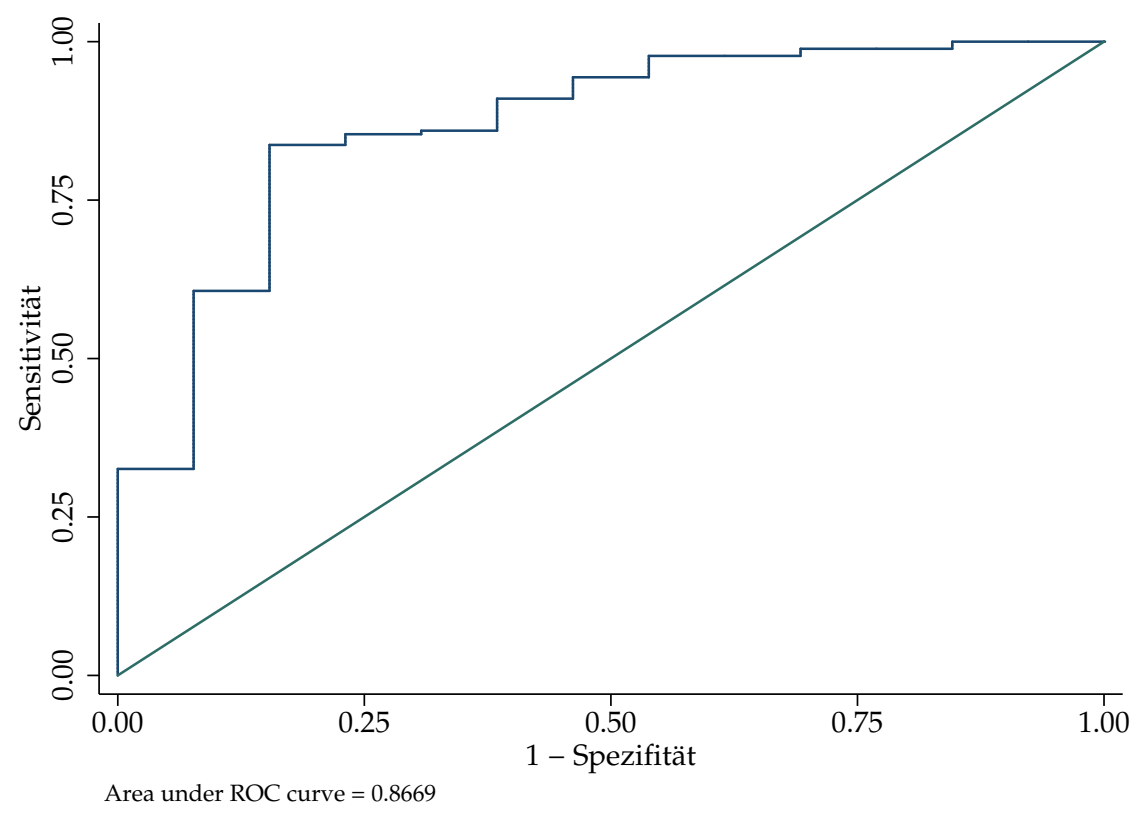

die bei einer rein zufälligen Zuordnung erreicht werden. Wie aus der Abbildung deutlich wird, erhöht sich mit steigendem Trennwert die Sensitivität des Modells, also die Anzahl der korrekt klassifizierten CLO-Transaktionen mit Downgrade. Jedoch geht dies zu Lasten der Falschpositiv-Rate, die ebenfalls mit der Höhe des unterstellten Trennwerts zunimmt. Den höchsten Anteil insgesamt korrekt klassifizierter Transaktionen (Trefferquote) von 94,24\% weist das Modell bei Trennwerten zwischen 56,72\% und 59,12\% sowie zwischen 68,95\% und 70,32\% auf. ${ }^{56}$ Liegt der Trennwert zwischen 56,72\% und 59,12\%, wird für insgesamt 185 Transaktionen eine Ratingherabstufung prognostiziert, von denen 176 korrekt und 9 falsch klassifiziert sind. Keine Ratingherabstufung wird für insgesamt nur 6 Transaktionen vorhergesagt, von denen tatsächlich 4 keinen Downgrade in 2009 erlitten. Demnach ergibt sich für diesen Trennwertbereich eine Sensitivität von 98,88\% und eine Spezifität von 30,77\%. Eine höhere Spezifität bei gleicher Trefferquote wird erreicht, wenn ein Trennwert zwischen 68,95\% und 70,32\% herangezogen wird. In diesem Bereich werden insgesamt 181 Transaktionen der Gruppe mit Ratingherabstufung zugeordnet, von denen tatsächlich 174 in 2009 einen Downgrade erfuhren. Keine Ratingherabstufung wird dagegen für 10 Transaktionen vorhergesagt, von denen 6 korrekt und 4 falsch klassifiziert sind. Diese Klassifikationsergebnisse korrespondieren mit einer Sensitivität von 97,75\% und einer Spezifität von 46,15\%. Aus den Klassifikationsergebnissen ist zu erkennen, dass das Regressionsmodell sehr gut in der Identifizierung herabgestufter Transaktionen ist, jedoch Schwächen in der Vorhersage nicht herabgestufter Transaktionen zeigt. Die „Area under a ROC curve (AUC)“ als Maß für die Modellgüte gibt an, dass das Regressionsmodell mit

$\overline{56}$ Tabelle 9 im Anhang zeigt die Klassifikationsmatrizen für diese Wertebereiche des Trennwerts. 
einer Wahrscheinlichkeit von 86,69\% die Downgrade-Wahrscheinlichkeit herabgestufter Transaktionen höher einschätzt als die nicht herabgestufter Transaktionen. ${ }^{57}$ Insgesamt ergibt sich somit eine gute Klassifikationsfähigkeit des Modells.

\subsection{Kritische Würdigung}

In der vorliegenden Studie werden die Leistungs- und Risikoanreize von CLO-Managern betrachtet, die durch eine performanceabhängige Vergütung hervorgerufen werden. CLOManager sind häufig auch an der Equity Tranche der von ihnen verwalteten Transaktion beteiligt, wodurch deren Anreize beeinflusst werden können. Gemäß der Argumentation für Hypothese 2 kann eine Beteiligung an der Equity Tranche einem CLO-Manager zusätzliche Anreize für ein sorgfältiges Screening und Monitoring geben und somit ein mögliches Leistungsanreizproblem abmildern. ${ }^{58}$ Andererseits kann eine Beteiligung an der Equity Tranche für den Manager auch Risikoanreize schaffen und ein mögliches Risk ShiftingProblem verstärken. ${ }^{59}$ Aufgrund der mangelnden Datenverfügbarkeit konnte in der vorliegenden Untersuchung die Bedeutung der Managerbeteiligung an der Equity Tranche für die Rating Performance nicht empirisch ermittelt werden. Da der Wert der Incentive Fee durch das von der Equity Tranche erzielte Ergebnis bestimmt wird, ist zu vermuten, dass in Analogie zur Incentive Fee eine Beteiligung an der Equity Tranche aufgrund des mit ihr verbundenen Risikoanreizproblems die Wahrscheinlichkeit einer Ratingherabstufung erhöht. Dieser Frage gilt es in weiteren empirischen Untersuchungen nachzugehen.

Wie bereits erwähnt, basieren die Ergebnisse der vorliegenden Studie auf einem Untersuchungszeitraum, in dem die Ratingagenturen aufgrund der Überarbeitung ihrer Ratingmethodik sämtliche CLO-Ratings einer Neubewertung unterzogen haben. Da eine Prognose zukünftiger Ratingherabstufungen voraussetzt, dass die von den Agenturen angewandte Ratingmethodik über die Zeit konstant ist, ist eine Vorhersage von DowngradeWahrscheinlichkeiten mittels der in der Untersuchung gewonnenen Ergebnisse nur eingeschränkt möglich. Des Weiteren ist das der Untersuchung zugrunde gelegte Jahr 2009 durch einen konjunkturellen Abschwung gekennzeichnet. Dies führt zu einer Verzerrung der Stichprobe, die einen Rückschluss auf die Downgrade-Wahrscheinlichkeiten von Arbitrage CLO-Transaktionen in wirtschaftlich besseren Zeiten nicht ohne Weiteres zulässt. Um die Allgemeingültigkeit der Erkenntnisse zu sichern, sind daher weitere empirische Untersuchungen mit längeren bzw. unterschiedlichen Beobachtungszeiträumen erforderlich.

\footnotetext{
${ }^{57}$ Siehe zur Bedeutung und Anwendung der AUC Hanley / McNeil (1982).

${ }^{58}$ Vgl. Garrison (2005), S. 18-19.

${ }^{59}$ Vgl. Garrison (2005), S. 20-22, und Scholz (2009), S. 32, 37 und 42 f.
} 


\section{Zusammenfassung und Ausblick}

Trotz der großen Bedeutung des Verbriefungsmarktes hat in der Literatur die Performance dieser Instrumente bisher nur wenig Beachtung gefunden. Ziel der vorliegenden Studie ist es, diese Lücke anhand einer Untersuchung der Erklärungsdeterminanten der Rating Performance europäischer Arbitrage CLO-Transaktionen im Jahr 2009 zu verkleinern. Die in die Untersuchung einbezogenen Erklärungsdeterminanten lassen sich in drei Kategorien unterteilen. Zum einen wird die Bedeutung der durch die Vergütung hervorgerufenen Leistungs- und Risikoanreize auf Seiten des CLO-Managers für die Rating Performance überprüft. Hierbei werden die Subordinated Fee und die Incentive Fee als performanceabhängige Bestandteile der Managervergütung herangezogen. Es wird gezeigt, dass die Existenz einer Incentive Fee die Wahrscheinlichkeit einer Ratingherabstufung erhöht. Dieser Befund bestätigt die Simulationsergebnisse von Scholz (2009), dass die Incentive Fee für CLO-Manager Anreize zu einer risikoreichen Anlagestrategie schafft. Das theoretisch hergeleitete Ergebnis von Garrison (2005), dass die Auswirkungen des aus der Incentive Fee resultierenden Leistungsanreizeffekts die des Risikoanreizeffekts übersteigen, wird somit nicht bestätigt. Für die Subordinated Fee hingegen kann kein Einfluss auf die Downgrade-Wahrscheinlichkeit einer CLO-Transaktion nachgewiesen werden. Des Weiteren wird die Bedeutung bestimmter Charakteristika von CLO-Managern für die Rating Performance untersucht. Dazu werden mehrere managerspezifische Faktoren zur Erklärung der Downgrade-Wahrscheinlichkeit identifiziert: die Erfahrung, die Größe, der Typ und der Unternehmenssitz eines CLO-Managers. Für die Erfahrung und die Größe wird ein negativer Zusammenhang mit der Wahrscheinlichkeit einer Ratingherabstufung festgestellt. Hinsichtlich des Managertyps zeigen von Investmentbanken gemanagte CLOTransaktionen im Untersuchungszeitraum eine im Durchschnitt geringere DowngradeWahrscheinlichkeit als Transaktionen, die von Multi-Asset Managern, Hedge Fonds oder Private Equity-Gesellschaften verwaltet werden. Gleichermaßen kann für Transaktionen US-amerikanischer Manager eine bessere Rating Performance als für Transaktionen europäischer Manager festgestellt werden. Schließlich wird der Erklärungsgehalt bestimmter transaktionsspezifischer Eigenschaften für die Rating Performance überprüft. Für die Qualität des Referenzportfolios, die Größe der Equity Tranche und das Transaktionsvolumen kann hierbei kein signifikanter Zusammenhang mit der Wahrscheinlichkeit einer Ratingherabstufung nachgewiesen werden.

Die Befunde dieser Studie geben erstmals einen Einblick in die Determinanten der Performance gemanagter Verbriefungstransaktionen. Der festgestellte negative Zusammenhang zwischen der Incentive Fee und der Performance belegt die Bedeutung von Anreizproblemen bei gemanagten Kreditverbriefungen. Anreizprobleme ergeben sich jedoch nicht nur auf Seiten der Asset Manager, sondern auf fast jeder Stufe des vor allem in den 
USA sehr weitgehend aufgespalteten Verbriefungsprozesses. ${ }^{60}$ Trotz dieser Probleme bringen Verbriefungen für Banken eine Vielzahl an Vorteilen mit sich. Sie stellen nicht nur ein Instrument zur Erzielung von Arbitragegewinnen dar, sondern insbesondere auch ein Instrument zur Refinanzierung und Steuerung ihrer Kreditportfolios. ${ }^{61}$ Zur Wiederbelebung und Stärkung des im Zuge der Finanzkrise ausgetrockneten Verbriefungsmarktes sind daher die mit den Problemen der asymmetrischen Informationsverteilung zusammenhängenden Risiken des Moral Hazard, der Adverse Selektion und der Kollusion durch geeignete Maßnahmen zu lösen. Lösungsvorschläge, wie etwa eine Begrenzung der Aufspaltung der Wertschöpfungskette oder die Korrektur verfehlter Anreizmechanismen auf den einzelnen Stufen des Kreditvergabeprozesses sowie der Prozesse des Risikotransfers am amerikanischen Markt, zielen dabei in die richtige Richtung. ${ }^{62}$

\footnotetext{
${ }^{60}$ Vgl. Ashcraft / Schuermann (2008), die die Friktionen zwischen den einzelnen beteiligten Parteien von Verbriefungen am US-amerikanischen Markt für Subprime-Darlehen detailliert beschreiben.

${ }^{61}$ Zur Bedeutung des Risikotransfers für die Bankensteuerung siehe Rudolph u. a. (2007), S. 165-170.

${ }^{62}$ Siehe Rudolph (2010), S. 128.
} 


\section{Literaturverzeichnis}

Adelson, Mark H. ; Bartlett, Elizabeth (2005): ABS Credit Migration Update, in: Journal of Structured Finance, Jg. 11, S. 51-63.

Adelson, Mark H. ; Sun, Yu ; Nikoulis, Panos ; Manzi, James (2002): ABS Credit Migrations, Nomura Fixed Income Research, März 2002.

Aminud, Yakov ; Lev, Baruch (1981): Risk Reduction as a Managerial Motive for Conglomerate Mergers, in: Bell Journal of Economics, Jg. 12, S. 605-617.

ARLT, Roland (2009): True Sale Securitisation unter besonderer Berücksichtigung der Rechtslage in Deutschland und Italien, Berlin 2009.

Ashcraft, Adam B. ; Schuermann, Til (2008): Understanding the Securitization of Subprime Mortgage Credit, Federal Reserve Bank of New York, Staff Report No. 318, März 2008.

Bamber, Donald (1975): The Area above the Ordinal Dominance Graph and the Area below the Receiver Operating Characteristic Graph, in: Journal of Mathematical Psychology, Jg. 12, S. 387-415.

BÄR, Hans P. (2000): Asset Securitisation: Die Verbriefung von Finanzaktiven als innovative Finanzierungstechnik und neue Herausforderungen für Banken, 3. Aufl., Bern et al. 2000.

Braun, Hendryk ; SCHMidt, Daniel (2005): Qualitative und quantitative Faktoren bei der Analyse von Asset-Backed-Securities, in: Praktiker-Handbuch Asset-Backed-Securities und Kreditderivate: Strukturen, Preisbildung, Anwendungsmöglichkeiten, aufsichtliche Behandlung, hrsg. von Josef Gruber, Walter Gruber und Hendryk Braun, Stuttgart 2005, S. 191-208.

Cantor, Richard ; Hu, Jian (2007): Deal Sponsor and Credit Risk of Asset-Backed and Mortgage-Backed Securities, Working Paper, Mai 2007.

CuchrA, Maciej Firla- (2005): Explaining Launch Spreads on Structured Bonds, Working Paper, University of Oxford, März 2005.

Cuchra, Maciej Firla- ; Jenkinson, Tim (2006): Security Design in the Real World: Why are Securitization Issues Tranched?, Working Paper, Januar 2006.

Delong, Elizabeth R. ; Delong, David M. ; Clarke-Pearson, Daniel L. (1988): Comparing the Areas under Two or More Correlated Receiver Operating Characteristic Curves: A Nonparametric Approach, in: Biometrics, Jg. 44, S. 837-845.

European Securitisation Forum (2008): ESF Securitisation Data Report Q4:2008.

European Securitisation Forum (2009): ESF Securitisation Data Report Q4:2009.

Fender, Ingo ; KIfF, John (2004): CDO Rating Methodology: Some Thoughts on Model Risk and its Implications, Bank for International Settlements, BIS Working Papers No. 163, November 2004. 
Fender, Ingo ; Mitchell, Janet (2009): Incentives and Tranche Retention in Securitisation: A Screening Model, Bank for International Settlements, BIS Working Papers No. 289, September 2009.

Fender, Ingo ; TARAshev, Nikola ; Zhu, Haibin (2008): Credit Fundamentals, Ratings and Value-at-Risk: CDOs versus Corporate Exposures, in: BIS Quarterly Review, März 2008, S. 87-101.

Fishelson-Holstine, Hollis (2004): The Role of Credit Scoring in Increasing Homeownership for Underserved Populations, Joint Center for Housing Studies, Working Paper Series BABC 04-12, Februar 2004.

Fitch (2007): European CLO Asset Managers - Survival of the Fittest, Structured Credit Europe Special Report, Januar 2007.

Fitch (2008a): Global Rating Criteria for Corporate CDOs, Global Structured Finance Criteria Report, April 2008.

Fitch (2008b): European CLO Asset Managers - Survival of the Fittest: The Return, CDO Asset Manager Europe Special Report, Juli 2008.

FiTCH (2010): European CLO Management Industry Update: Brighter Prospects For Select Top-Tier Managers, CDO Asset Managers Europe Special Report, Juni 2010.

Franke, Günter ; Herrman, Markus ; Weber, Thomas (2007): Information Asymmetries and Securitization Design, Working Paper, Dezember 2007.

Franke, Günter ; WeBer, Thomas (2007): Wie werden Collateralized Debt ObligationTransaktionen gestaltet?, in: Zeitschrift für betriebswirtschaftliche Forschung, Sonderheft 57/07, S. 95-123.

Franke, Günter ; Weber, Thomas (2009): Optimal Tranching in CDO-Transactions, Working Paper, Februar 2009.

Gan, Yingjin H. ; MAYER, Christopher (2007): Agency Conflicts, Asset Substitution, and Securitization, Working Paper, Mai 2007.

Garrison, Kedran R. (2005): Manager Incentives in Collateralized Debt Obligations, Working Paper, August 2005.

Greene, William H. (2000): Econometric Analysis, Upper Saddle River 2000.

Güttler, Andre ; Hommel, Ulrich ; Reichert, Julia (2010): The Influence of Sponsor, Servicer and Underwriter Characteristics on RMBS Performance, Working Paper, April 2010.

Hanley, James A. ; MCNeil, Barbara J. (1982): The Meaning and Use of the Area under a Receiver Operating Characteristic (ROC) Curve, in: Radiology, Jg. 143, S. 29-36.

Hirshleifer, David ; Thakor, Anjan V. (1992): Managerial Conservatism, Project Choice, and Debt, in: Review of Financial Studies, Jg. 5, S. 437-470.

Holmström, Bengt ; Ricart I Costa, Joan (1986): Managerial Incentives and Capital Management, in: Quarterly Journal of Economics, Jg. 101, S. 835-860. 
InNeS, Robert D. (1990): Limited Liability and Incentive Contracting with Ex-ante Action Choices, in: Journal of Economic Theory, Jg. 52, S. 45-67.

Jobst, Andreas (2002): Collateralised Loan Obligations (CLOs) - A Primer, Center for Financial Studies, CFS Working Paper No. 2002/13, Dezember 2002.

Keller, Joachim (2008): Agency Problems in Structured Finance - A Case Study of European CLOs, National Bank of Belgium, Working Paper No. 137, August 2008.

Keys, Benjamin J. ; MukherJee, Tanmoy ; Seru, Amit ; Vig, Vikrant (2009): Financial Regulation and Securitization: Evidence from Subprime Loans, in: Journal of Monetary Economics, Jg. 56, S. 700-720.

Kothari, Vinod (2006): Securitization: The Financial Instrument of the Future, Singapur 2006.

Lassalvy, Laurent ; Lautard, Frédéric (2010): How Well Did European CLO Managers Avoid Default?, in: CLO Interest, hrsg. von Algis Remeza und Jerry Gluck, Moody's Investors Service, März 2010, S. 15-16.

Micu, Marian ; Remolona, Eli M. ; Wooldridge, Philip D. (2004): Preiseffekte von Rating-Meldungen: Untersuchungen am Markt für Credit Default Swaps, in: BIZQuartalsbericht, Juni 2004, S. 63-75.

Moody's Investors Service (2009a): Announcement: Moody's Updates Key Assumptions for Rating CLOs, Global Credit Research, Februar 2009.

Moody's Investors Service (2009b): Rating Action: Moody's Takes Rating Action on 71 Notes Issued by 21 CLO Transactions, Global Credit Research, 17. März 2009.

Moody's Investors Service (2009c): Rating Action: Moody's Takes Rating Action on 99 Notes Issued by 30 CLO Transactions, Global Credit Research, 18. März 2009.

Moody's Investors Service (2009d): Rating Action: Moody's Takes Rating Action on 227 Notes Issued by 66 CLO Transactions, Global Credit Research, 19. März 2009.

Moody's Investors Service (2009e): Rating Action: Moody's Takes Rating Action on 57 Notes Issued by 18 CLO Transactions, Global Credit Research, 20. März 2009.

Moody's Investors SERViCE (2009f): Moody's Approach to Rating Collateralized Loan Obligations, Rating Methodology, August 2009.

Moody's Investors Service (2010): Annual Sector Review (2009): Global CLOs, Special Report, März 2010.

Newman, Daniel ; Fabozzi, Frank J. ; Lucas, Douglas J. ; Goodman, Laurie S. (2008): Empirical Evidence on CDO Performance, in: Journal of Fixed Income, Jg. 18, S. 32-40.

Rudolph, Bernd (2010): Die internationale Finanzkrise als Anstoß für Weiterentwicklungen im Risikocontrolling der Banken und für Reformen in der Bankregulierung, in: Zeitschrift für betriebswirtschaftliche Forschung, Sonderheft 61/10, S. 122-149.

Rudolph, Bernd ; Hofmann, Bernd ; Schaber, Albert ; Schäfer, Klaus (2007): Kreditrisikotransfer - Moderne Instrumente und Methoden, Berlin et al. 2007. 
SchaBer, Albert (2009): Collateralized Debt Obligations: First Loss Piece Retention, Combination Notes, and Tranching, Frankfurt am Main et al. 2009.

Schiefer, Dirk (2008): Collateralized Debt Obligations (CDOs): Eine empirische Analyse der Bonitätsrisikoprämie auf Finanzmärkten, Bad Soden/Ts. 2008.

Scholz, Julia (2009): Collateralized Debt Obligations: Anreizprobleme im Rahmen des Managements von CDOs, Münchener Wirtschaftswissenschaftliche Beiträge (BWL) 2009-15, September 2009.

StAndard \& PoOR's (2002): Global Cash Flow and Synthetic CDO Criteria, März 2002.

Standard \& Poor's (2009a): Update to Global Methodologies and Assumptions for Corporate Cash Flow and Synthetic CDOs, September 2009.

Standard \& Poor's (2009b): CDOs: Supplementary Assumptions Relating To CDO Evaluator Version 5.0: The Corporate Credit Rating Transition Matrix, November 2009.

Waschbusch, Gerd (1998): Asset Backed Securities - eine moderne Form der Unternehmensfinanzierung, in: Zeitschrift für Bankrecht und Bankwirtschaft, Jg. 10, S. 408-419.

Wooldridge, Jeffrey M. (2009): Introductory Econometrics: A Modern Approach, Mason 2009. 


\section{Anhang}

Tabelle 6: Beschreibung der in der Studie verwendeten Variablen.

\begin{tabular}{|c|c|}
\hline Variable & Beschreibung \\
\hline \multicolumn{2}{|l|}{ Rating Performance: } \\
\hline DOWNGRADE & $=1$, falls mindestens eine Tranche der CLO-Transaktion in 2009 herabgestuft wurde \\
\hline \multicolumn{2}{|l|}{ Managervergütung: } \\
\hline $\begin{array}{l}\text { SUBFEE } \\
\text { INCFEE }\end{array}$ & $\begin{array}{l}\text { Höhe der Subordinated Fee (in \%) } \\
=1 \text {, falls eine Incentive Fee im Management Agreement der CLO-Transaktion } \\
\text { vereinbart ist }\end{array}$ \\
\hline \multicolumn{2}{|l|}{ Managercharakteristika: } \\
\hline $\begin{array}{l}\text { DEALS } \\
\text { lnDEALS } \\
\text { AUM } \\
\text { lnAUM } \\
\text { LF SPEZIALIST } \\
\text { LF BANK } \\
\text { MULTI-ASSET } \\
\text { HEDGE FOND } \\
\text { PRIVATE EQUITY } \\
\text { US }\end{array}$ & $\begin{array}{l}\text { Anzahl der bisher vom CLO-Manager am europäischen Markt verwalteten CLO- } \\
\text { Transaktionen (gemessen zum Emissionszeitpunkt) } \\
\text { natürlicher Logarithmus der Variable DEALS } \\
\text { Volumen des vom CLO-Manager verwalteten Vermögens in europäischen CLO- } \\
\text { Transaktionen (in Mio. EUR, gemessen zum Emissionszeitpunkt) } \\
\text { natürlicher Logarithmus der Variable AUM } \\
=1 \text {, falls CLO-Manager ein Leverage Finance Spezialist ist } \\
=1 \text {, falls CLO-Manager eine Investmentbank (Leverage Finance Abteilung) ist } \\
=1 \text {, falls CLO-Manager ein Multi-Asset Manager ist } \\
=1 \text {, falls CLO-Manager ein Hedge Fond ist } \\
=1 \text {, falls CLO-Manager eine Private Equity-Gesellschaft ist } \\
=1 \text {, falls Sitz des CLO-Managers USA ist }\end{array}$ \\
\hline \multicolumn{2}{|l|}{ Transaktionscharakteristika: } \\
\hline $\begin{array}{l}\text { WARF } \\
\operatorname{lnWARF} \\
\text { FLP } \\
\text { SIZE } \\
\text { lnSIZE }\end{array}$ & $\begin{array}{l}\text { durchschnittlicher Ratingfaktor der CLO-Transaktion } \\
\text { natürlicher Logarithmus der Variable WARF } \\
\text { Größe der Equity Tranche (in \%) } \\
\text { Volumen der CLO-Transaktion (in Mio. EUR) } \\
\text { natürlicher Logarithmus der Variable SIZE }\end{array}$ \\
\hline \multicolumn{2}{|l|}{ Emissionsjahre: } \\
\hline $\begin{array}{l}\text { J2001, J2002, J2003, J2004, } \\
\text { J2005, J2006, J2007, J2008 } \\
\text { J0102 } \\
\text { J0304 } \\
\text { J0506 } \\
\text { J0708 }\end{array}$ & $\begin{array}{l}=1 \text {, falls CLO-Transaktion im Jahr 2001, 2002, 2003, 2004, 2005, 2006, } 2007 \text { bzw. } \\
2008 \text { emittiert wurde } \\
=1 \text {, falls CLO-Transaktion im Jahr } 2001 \text { oder } 2002 \text { emittiert wurde } \\
=1 \text {, falls CLO-Transaktion im Jahr } 2003 \text { oder } 2004 \text { emittiert wurde } \\
=1 \text {, falls CLO-Transaktion im Jahr } 2005 \text { oder } 2006 \text { emittiert wurde } \\
=1 \text {, falls CLO-Transaktion im Jahr } 2007 \text { oder } 2008 \text { emittiert wurde }\end{array}$ \\
\hline
\end{tabular}


Tabelle 7: Zur Berechnung der Variable WARF verwendete Ratingfaktoren für die Bonitätseinstufungen der Ratingagenturen Moody's, Standard \& Poor's und Fitch.

\begin{tabular}{|c|c|c|c|c|c|}
\hline Ratingfaktor & $\begin{array}{l}\text { Rating } \\
\text { Moody's }\end{array}$ & $\begin{array}{c}\text { Rating } \\
\text { S \& P / Fitch }\end{array}$ & Ratingfaktor & $\begin{array}{l}\text { Rating } \\
\text { Moody's }\end{array}$ & $\begin{array}{c}\text { Rating } \\
\text { S \& P / Fitch }\end{array}$ \\
\hline 1 & Aaa & AAA & 940 & $\mathrm{Ba1}$ & $\mathrm{BB}+$ \\
\hline 10 & Aa1 & $\mathrm{AA}+$ & 1.350 & $\mathrm{Ba} 2$ & $\mathrm{BB}$ \\
\hline 20 & $\mathrm{Aa} 2$ & $\mathrm{AA}$ & 1.766 & $\mathrm{Ba} 3$ & $\mathrm{BB}-$ \\
\hline 40 & $\mathrm{Aa} 3$ & $\mathrm{AA}-$ & 2.220 & B1 & $\mathrm{B}+$ \\
\hline 70 & A1 & $\mathrm{A}+$ & 2.720 & B2 & B \\
\hline 120 & $\mathrm{~A} 2$ & $\mathrm{~A}$ & 3.490 & B3 & $\mathrm{B}-$ \\
\hline 180 & A3 & $\mathrm{A}-$ & 4.770 & Caa1 & $\mathrm{CCC}+$ \\
\hline 260 & Baa1 & $\mathrm{BBB}+$ & 6.500 & Caa2 & $\mathrm{CCC}$ \\
\hline 360 & $\mathrm{Baa} 2$ & $\mathrm{BBB}$ & 8.070 & Caa3 & $\mathrm{CCC}-$ \\
\hline 610 & Baa3 & $\mathrm{BBB}-$ & 10.000 & $\mathrm{Ca}, \mathrm{C}$ & $\mathrm{CC}, \mathrm{C}$ \\
\hline
\end{tabular}

Tabelle 8: Korrelationsmatrix der erklärenden Variablen. Die Tabelle berichtet die Bravais-PearsonKorrelationskoeffizienten der metrisch skalierten Variablen. Signifikante Korrelationen sind mit Asterisken gekennzeichnet, wobei * signifikant auf dem 1\%-Niveau bedeutet.

\begin{tabular}{lrccccc}
\hline & SUBFEE & $\operatorname{lnDEALS}$ & $\operatorname{lnAUM}$ & $\ln$ ARRF & FLP & $\operatorname{lnSIZE}$ \\
\hline SUBFEE & 1,000 & & & & & \\
lnDEALS & $-0,019$ & 1,000 & & & & \\
lnAUM & 0,017 & $0,968^{*}$ & 1,000 & & & \\
lnWARF & $-0,007$ & $-0,159$ & $-0,142$ & 1,000 & 1,000 & 1,000 \\
FLP & $-0,086$ & $-0,055$ & $-0,037$ & $0,850^{*}$ & $-0,030$ & \\
lnSIZE & 0,024 & $0,262^{*}$ & $0,430^{*}$ & 0,006 & & \\
\hline
\end{tabular}

Tabelle 9: Klassifikationsergebnisse des Probitmodells für verschiedene Trennwerte. Es werden die Schätzergebnisse der Modellspezifikation I der Tabelle 4 verwendet.

\begin{tabular}{|c|c|c|c|}
\hline \multirow[b]{3}{*}{ Beobachtet } & \multicolumn{3}{|c|}{ PANEL A: Trennwert zwischen $56,72 \%$ und $59,12 \%$} \\
\hline & \multicolumn{2}{|c|}{ Vorhergesagt } & \multirow[b]{2}{*}{ Summe } \\
\hline & Downgrade & Kein Downgrade & \\
\hline Downgrade & 176 & 2 & 178 \\
\hline Kein Downgrade & 9 & 4 & 13 \\
\hline \multirow[t]{3}{*}{ Summe } & 185 & 6 & 191 \\
\hline & \multicolumn{3}{|c|}{ PANEL B: Trennwert zwischen $68,95 \%$ und $70,32 \%$} \\
\hline & \multicolumn{2}{|c|}{ Vorhergesagt } & \\
\hline Beobachtet & Downgrade & Kein Downgrade & Summe \\
\hline Downgrade & 174 & 4 & 178 \\
\hline Kein Downgrade & 7 & 6 & 13 \\
\hline Summe & 181 & 10 & 191 \\
\hline
\end{tabular}

